\title{
THE ASYMPTOTIC BEHAVIOR OF SOLUTIONS OF SYS- TEMS OF VOLTERRA INTEGRAL EQUATIONS
}

BY

\section{ALFRED HORN}

Introduction. Although the asymptotic properties of solutions of differential equations have been intensively studied, the corresponding question for integral equations seems to have been neglected. This is surprising because most investigations of the asymptotic behavior of solutions of differential equations begin by converting the differential equation into an integral equation (with a special kernel).

In this paper we obtain an asymptotic series for the solution of the system of Volterra integral equations

$$
u_{i}(x, y, \lambda)=\sum_{j=1}^{n} \lambda \int_{y}^{x} K_{i j}(x, t, \lambda) u_{j}(t, y, \lambda) d t+f_{i}(x, y, \lambda) .
$$

The kernels $K_{i j}$ and functions $f_{i}$ are assumed to have asymptotic expansions of the form

$$
\begin{aligned}
K_{i j}(x, y, \lambda) & =K_{i j}^{0}(x, y)+K_{i j}^{1}(x, y) / \lambda+\cdots, \\
f_{i}(x, y, \lambda) & =f_{i}^{0}(x, y)+f_{i}^{1}(x, y) / \lambda+\cdots,
\end{aligned}
$$

valid in certain regions of the complex $\lambda$-plane. Our fundamental assumption is that the characteristic roots of the matrix $\left(K_{y}^{0}(x, x)\right)$ are distinct and different from 0 for all values of $x$ in the closed interval $[\alpha, \beta]$.

Consider the special case of a single differential equation

$$
\frac{d}{d x} u(x, \lambda)=\lambda K(x) u(x, \lambda)+f(x) .
$$

This has as solution

$$
u(x, \lambda)=u(\alpha) \exp \left(\lambda \int_{\alpha}^{x} K(s) d s\right)+\int_{\alpha}^{x} \exp \left(\lambda \int_{t}^{x} K(s) d s\right) f(t) d t .
$$

If $K(x) \neq 0$ in $[\alpha, \beta]$ we may integrate by parts to obtain the series

$$
u(x, \lambda)=\exp \left(\lambda \int_{\alpha}^{x} K(s) d s\right)\left(F^{0}+F^{1} / \lambda+\cdots\right)+g^{1}(x) / \lambda+\cdots+r(x, \lambda) / \lambda^{k},
$$

where $F^{0}=u(\alpha), \quad F^{1}=f(\alpha) / K(\alpha), g^{1}(x)=-f(x) / K(x)$, and $r(x, \lambda)=\int_{\alpha}^{x} \exp$ $\left(\lambda \int_{i}^{x} K(s) d s\right) h(t) d t$, where $h$ is continuous if $K$ and $f$ are of class $C^{k}$. If $K(x)$ is

Presented to the Society, November 30, 1946; received by the editors December 2, 1946. 
negative in $[\alpha, \beta]$ the remainder $r(x, \lambda) / \lambda^{k}$ is in absolute value not greater than const. $/|\lambda|^{k}$ for all $x$ in $[\alpha, \beta]$ and all $\lambda$ in the right half-plane. In the case of a system of $n$ linear differential equations

$$
\frac{d}{d x} u_{i}(x, \lambda)=\sum_{j=1}^{n} \lambda K_{i j}(x, \lambda) u_{j}(x, \lambda)+f_{i}(x, \lambda)
$$

one could apply Birkhoff's results (see [1, pp. 71-87]) (1) on the behavior of solutions of the homogeneous system and integration by parts to obtain an asymptotic series of the form

$$
\begin{aligned}
u_{i}(x, \lambda)= & \exp \left(\lambda \int_{\alpha}^{x} \gamma_{1}(s) d s\right)\left(F_{i 1}^{0}+F_{i 1}^{1} / \lambda+\cdots\right)+\cdots \\
& +\exp \left(\lambda \int_{\alpha}^{x} \gamma_{n}(s) d s\right)\left(F_{i n}^{0}+F_{i n}^{1} / \lambda+\cdots\right) \\
& +g_{i}^{1}(x) / \lambda+g^{2}(x) / \lambda^{2}+\cdots,
\end{aligned}
$$

where the $\gamma_{i}(x)$ are the characteristic roots of the matrix $\left(K_{v}^{0}(x)\right)$. This is exactly the type of expansion we obtain in the more general case (1). Thus the solutions of Volterra equations exhibit no new features in asymptotic behavior as compared. with solutions of differential equations. The only difference is in the law of formation of the coefficients $F_{u}^{k}, g_{v}^{k}$. In the case of integral equations the $F_{v}^{k}$ are functions of $x$ and $y$ determined by solving first order linear differential equations and the $g_{i}^{k}$ are determined by solving systems of Volterra equations of the first kind with the kernel matrix $\left(K_{i j}^{0}(x, y)\right)$.

In $\$ 2$ we derive the formal series solutions of (1). The asymptotic character of this formal series is investigated in $\S 3$. We do not make use of Birkhoff's theorem in this analysis. $\$ 4$ is concerned with cases in which certain terms of the expansion vanish. For example when $f_{i}(x, y, \lambda)=K_{i j}(x, y, \lambda)$ the solution of (1) is the $j$ th column of the resolvent matrix. In this case the terms $g_{i}^{1}$ are identically 0 , and necessary and sufficient conditions for higher order terms to vanish are derived. In $\$ 5$ we apply our results to integrodifferential equations and also investigate the approach of the solution of the equation

$$
\theta(x)=\lambda \int_{\alpha}^{x} \Gamma(x, t) \theta(t) d t+\lambda \phi(x)
$$

to the solution of the equation of the first kind

$$
0=\int_{\alpha}^{x} \Gamma(x, t) \theta(t) d t+\phi(x),
$$

(1) Numbers in brackets refer to the references cited at the end of the paper. 
under the assumption that the kernel $\Gamma$ has a definite order. It turns out that the solution of (2) can approach the solution of (3) only if the order is not greater than 2 .

It is believed that most of the results are new even in the case $n=1$, and $K_{11}$ and $f_{1}$ independent of $\lambda$. Volterra [6, pp. 241-243] derived what amounts to the first two terms of the expansion in this case, with real-valued functions and $\lambda$ approaching infinity along the real axis. Peres $[4$, pp. 26-30] gave another proof of Volterra's results based on the theory of functions of composition. Trjitzinsky [5, pp. 40-44] obtained a bound for the solution of the integro-differential equation considered in $\$ 5$ in the case where the characteristic roots of the differential operator are not assumed distinct.

This paper constitutes a generalization of my Ph.D. thesis written at the University of California, and I wish to acknowledge helpful suggestions of Professor G. C. Evans in the course of that study.

1. Notation and definitions. We shall use the following definitions and conventions.

Definition 1.1. Let $T$ be the set of points $(x, y)$ such that $\alpha \leqq y \leqq x \leqq \beta$, and let $I$ be the interval $\alpha \leqq x \leqq \beta$. Here $\alpha$ and $\beta$ are finite.

Definition 1.2. A function on $T$ (or $I$ ) is said to be of class $C^{p}$ if its derivatives of order $p$ are continuous on $T$ (or $I$ ), $p \geqq 0$.

We shall be concerned with functions on $T$ or $I$ which carry points into complex-valued matrices, vectors, or numbers. To distinguish between the three types of functions we shall use Italic capitals, Italic lower case, and Greek letters respectively. The order of the matrices and vectors is fixed throughout and we denote it by $n$. The case $n=1$ is not excluded, and in this case all statements about non-diagonal terms, and so on, are to be disregarded. All summations in which no limits appear for the summation index shall run from 1 to $n$. Thus $\sum_{m \neq i} a_{m}=a_{1}+\cdots+a_{i-1}+a_{i+1}+\cdots+a_{n}$.

The $i, j$ element of $A(x)$ is denoted by $A(x)_{i j}$ or $A_{i j}(x)$. Similarly $e_{i}(x)$ or $e(x)_{i}$ is used to denote the $i$ th component of $e(x)$. Occasionally we shall use $\left(\eta_{i j}(x)\right)$ to denote the matrix whose $i, j$ element is $\eta_{i j}(x)$.

Definition 1.3. If $A$ and $B$ are matrix functions on $T$ then $A B$ is the matrix function on $T$ such that $A B(x, y)=A(x, y) B(x, y)$, the ordinary matrix product. If $A$ and $B$ are on $I, A B$ is defined in a similar way.

Definition 1.4. If $A$ is a matrix function and $u$ is a vector function, then $A u$ is the vector function such that $A u(x, y)=A(x, y) u(x, y)$. The right side is a vector whose $i$ th component is $\sum_{j} A_{i j}(x, y) u_{j}(x, y)$.

Definition 1.5. If $A$ and $B$ are continuous on $T$, then $A * B$ is the matrix function on $T$ such that $A * B(x, y)=\int_{v}^{x} A(x, t) B(t, y) d t$. This Volterra product is associative. We define $A^{1^{*}}=A$, and $A^{p^{*}}=A * A^{(p-1)^{*}}$ for $p \geqq 2$.

Definition 1.6. If $A$ and $u$ are continuous on $T$, then $A * u$ is the vector function on $T$ such that $A * u(x, y)=\int_{v}^{x} A(x, t) u(t, y) d t$. We have $A *(B * u)$ $=(A * B) * u$. 
We shall use the notation $D_{1} A$ or $D_{2} A$ to denote the matrix function whose value at the point $(x, y)$ is the derivative of $A$ with respect to its first or second variable at the point $(x, y)$. A similar notation is used for derivatives of vector functions and ordinary functions. For example if $\gamma(x, y)=x^{2}+x y^{3}$, then $D_{1} \gamma(t, x)=2 t+x^{3}$.

We now summarize the basic part of the theory of systems of Volterra integral equations. The proofs may be found for example in [2].

Let $F(x, y, \lambda)$ be defined for $(x, y)$ in $T$ and $\lambda$ in some set $Z$ in the complex plane, and suppose $F$ is continuous on $T$ for each $\lambda$ in $Z$. Then the series $\sum_{i=1}^{\infty} \lambda^{i-1} F_{i}^{*}(x, y, \lambda)$ converges uniformly in $T$ for each $\lambda$ in $Z$. If we let $G(x, y, \lambda)$ be this sum, then $G$ is continuous on $T$ for each $\lambda$ in $Z$, and it is easily seen that $G-F=\lambda F * G=\lambda G * F$. Using these relations, one can show that if $F$ is of class $C^{p}$ on $T$, then $G$ is also of class $C^{p}$ on $T$ for each $\lambda$ in $Z$. $G$ is called the resolvent of $F$. If $f$ is continuous on $I$ for each $\lambda$ in $Z$, the solution of the system $u=\lambda F * u+f$ is given by $u=f+\lambda G * f$.

Definitions, lemmas, and theorems are numbered consecutively in each section.

2. Formal developments. Consider the system

$$
u(x, y, \lambda)=\lambda \cdot \int_{y}^{x} K(x, t, \lambda) u(t, y, \lambda) d t+f(x, y, \lambda),
$$

in which

$$
\begin{gathered}
K(x, y, \lambda)=\sum_{i=0}^{k} \lambda^{-i} K^{i}(x, y)+\lambda^{-k-1} K^{\prime}(x, y, \lambda), \\
f(x, y, \lambda)=\sum_{i=0}^{k} \lambda^{-i} f^{i}(x, y)+\lambda^{-k-1} f^{\prime}(x, y, \lambda)
\end{gathered}
$$

for $(x, y)$ in $T$ and $\lambda$ in a certain unbounded set $Z$ of the complex plane. The existence of bounds for $K^{\prime}$ and $f^{\prime}$ is not needed in this section. We assume $K^{i}$ and $f^{i}$ are of class $C^{k+1-i}$ on $T, 0 \leqq i \leqq k$, and that for each $\lambda$ in $Z, K^{\prime}$ and $f^{\prime}$ are continuous on $T$. Also we suppose that the characteristic roots $\gamma_{1}(x), \cdots, \gamma_{n}(x)$ of $K^{0}(x, x)$ are distinct and different from 0 for each $x$ in $I$. Finally we assume $k \geqq 1$ in the sense that although $K^{1}, \cdots, K^{k}$ and $K^{\prime}$ may be identically zero, we wish $K^{0}$ to be at least of class $C^{2}$.

Let $C(x)$ be a matrix whose $p$ th column $c^{p}(x)$ is a characteristic vector corresponding to $\gamma_{p}(x)$. The $\gamma_{i}$ are of class $C^{k+1}$ and it is easy to show that we may take $C$ to be of class $C^{k+1}$. The vectors $c^{p}(x)$ are linearly independent and hence $C(x)$ is nonsingular for each $x$ in $I$. If we make the change of variable $u(x, y, \lambda)=C(x) v(x, y, \lambda)$, and set $R^{i}(x, y)=C(x)^{-1} K^{i}(x, y) C(y), R^{\prime}(x, y, \lambda)$ $=C(x)^{-1} K^{\prime}(x, y, \lambda) C(y), h^{i}(x, y)=C(x)^{-1} f^{i}(x, y), h^{\prime}(x, y, \lambda)=C(x)^{-1} f^{\prime}(x, y, \lambda)$, then (2.1) takes the form 


$$
v(x, y, \lambda)=\lambda \int_{y}^{x} R(x, t, \lambda) v(t, y, \lambda) d t+h(x, y, \lambda)
$$

where

$$
\begin{aligned}
& R(x, y, \lambda)=\sum_{i=0}^{k} \lambda^{-i} R^{i}(x, y)+\lambda^{-k-1} R^{\prime}(x, y, \lambda), \\
& h(x, y, \lambda)=\sum_{i=0}^{k} \lambda^{-i} h^{i}(x, y)+\lambda^{-k-1} h^{\prime}(x, y, \lambda) .
\end{aligned}
$$

By definition of $C(x)$ we have $R^{0}(x, x)=C(x)^{-1} K^{0}(x, x) C(x)=\left(\gamma_{i}(x) \delta_{i j}\right)$. For reference we collect our hypotheses for the system (2.3).

Hypotheses A. (i) $R^{i}$ and $h^{i}$ are of class $C^{k+1-i}, 0 \leqq i \leqq k, k \geqq 1$; (ii) $R^{\prime}$ and $h^{\prime}$ are continuous on $T$ for each $\lambda$ in $Z$; (iii) $R^{0}(x, x)=\left(\gamma_{i}(x) \delta_{i j}\right)$; (iv) $\gamma_{i}(x) \neq \gamma_{j}(x)$ for $x \in I, i \neq j, 1 \leqq i \leqq n, 1 \leqq j \leqq n ;(v) \gamma_{i}(x) \neq 0$ for each $x$ in $I$, $1 \leqq i \leqq n$.

Definition 2.1. If $R$ and $F$ are matrix functions on $T$, we define $R: F(x, t, y)=R(x, t) F(t, y)$ for $\alpha \leqq y \leqq t \leqq x \leqq \beta(2)$.

Definition 2.2. If $G(x, t, y)$ is defined for $\alpha \leqq y \leqq t \leqq x \leqq \beta$, we define over this same range the following functions:

$$
\begin{array}{ll}
D^{0}(G)(x, z, y)=G(x, z, y), & \\
D^{1}(G)(x, z, y) & =D(G)(x, z, y)=\frac{\partial}{\partial t}\left[G(x, t, y) R^{0}(t, t)^{-1}\right]_{t=z}, \\
D^{p}(G)(x, z, y) & =D\left(D^{p-1}(G)\right)(x, z, y), \quad p \geqq 2 .
\end{array}
$$

The operator $D$ is not to be confused with the differentiation operators $D_{1}, D_{2}$ defined in $\$ 1$. For future reference we calculate the diagonal terms of $D\left(R^{0}: F\right)(x, x, y)$. These are given by

$$
\begin{aligned}
{\left[D\left(R^{0}: F\right)(x, x, y)\right]_{i i}=} & \sum_{m} \frac{\partial}{\partial t}\left[R_{i m}^{0}(x, t) F_{m i}(t, y) / \gamma_{i}(t)\right]_{t=x} \\
= & \sum_{m} R_{i m}^{0}(x, x)\left[D_{1} F_{m i}(x, y) / \gamma_{i}(x)\right. \\
& \left.-\frac{d}{d x} \gamma_{i}(x) F_{m i}(x, y) / \gamma_{i}(x)^{2}\right] \\
& +\sum_{m} F_{m i}(x, y) D_{2} R_{i m}^{0}(x, x) / \gamma_{i}(x)
\end{aligned}
$$

(2) If $f$ is a vector function, $R: f$ is defined in the same way. 


$$
\begin{aligned}
= & D_{1} F_{i i}(x, y)-\frac{d}{d x} \gamma_{i}(x) F_{i i}(x, y) / \gamma_{i}(x) \\
& +\sum_{m} F_{m i}(x, y) D_{2} R_{i m}^{0}(x, x) / \gamma_{i}(x) \\
= & D_{1} F_{i i}(x, y)+F_{i i}(x)\left[-\frac{d}{d x} \gamma_{i}(x)+D_{2} R_{i i}^{0}(x, x)\right] / \gamma_{i}(x) \\
& +\sum_{m \neq i} F_{m i}(x, y) D_{2} R_{i m}^{0}(x, x) / \gamma_{i}(x) \\
= & D_{1} F_{i i}(x, y)-F_{i i}(x, y) D_{1} R_{i i}^{0}(x, x) / \gamma_{i}(x) \\
& +\sum_{m \neq i} F_{m i}(x, y) D_{2} R_{i m}^{0}(x, x) / \gamma_{i}(x) .
\end{aligned}
$$

In the last step we have used the fact that $D_{1} R_{\dot{H}}^{0}(x, x)+D_{2} R_{\sharp y}^{0}(x, x)=d \gamma_{i}(x) / d x$.

Definition 2.3. $e(x, y)$ is the vector such that $e_{i}(x, y)=\exp \left(\lambda \int_{i}^{x} \gamma_{i}(s) d s\right)$.

LEMma 2.4. If $R$ and $F$ are of class $C^{r}$ on $T, r \geqq 1$, then

$$
\begin{aligned}
\lambda \int_{y}^{x} R(x, t) F(t, y) e(t, y) d t= & \sum_{j=0}^{r-1}(-1)^{i-j}\left[D^{i}(R: F)(x, t, y) R^{0}(t, t)^{-1} e(t, y)\right]_{t=y}^{t=x} \\
& +(-1)^{r-\lambda-(r-1)} \int_{y}^{x} D^{r}(R: F)(x, t, y) e(t, y) d t .
\end{aligned}
$$

Proof. Obviously $D_{1} e(x, y)=\lambda R^{0}(x, x) e(x, y)$. Integration by parts gives

$$
\begin{aligned}
\lambda \int_{y}^{x} R(x, t) F(t, y) e(t, y) d t= & {\left[R(x, t) F(t, y) R^{0}(t, t)^{-1} e(t, y)\right]_{t=y}^{t=x} } \\
& -\int_{y}^{x} \frac{\partial}{\partial t}\left[R(x, t) F(t, y) R^{0}(t, t)^{-1}\right] e(t, y) d t \\
= & {\left[D^{0}(R: F)(x, t, y) R^{0}(t, t)^{-1} e(t, y)\right]_{t=y}^{t=x} } \\
& -\int_{y}^{x} D^{1}(R: F)(x, t, y) e(t, y) d t .
\end{aligned}
$$

Applying this process $r-1$ more times, we obtain our result.

Lemma 2.5. If $a$ is of class $C^{r}, 1 \leqq r \leqq k+1$, and if $a(y, y) \equiv 0$, then the system

$$
\int_{y}^{x} R^{0}(x, t) g(t, y) d t=u(x, y)
$$

has a unique solution $g$ of class $C^{r-1}$.

Proof. The system (2.6) with $a(y, y) \equiv 0$ is equivalent to the system 


$$
R^{0}(x, x) g(x, y)+\int_{y}^{x} D_{1} R^{0}(x, t) g(t, y) d t=D_{1} a(x, y),
$$

which has the solution $g(x, y)=b(x, y)+\int_{y}^{x} S(x, t, 1) b(t, y) d t$, where $b(x, y)$ $=R^{0}(x, x)^{-1} D_{1} a(x, y)$ and $S$ is the resolvent of the kernel whose value at $(x, y)$ is $R^{0}(x, x)^{-1} D_{1} R^{0}(x, y)$. But $S$ is of class $C^{k}$ (see $\$ 1$ ), while $b$ is of class $C^{r-1}$.

THEOREM 2.6. Under Hypotheses A, there exist functions $F^{q}$ and $g^{a}$ of class $C^{k+1-q}$ on $T$ such that if we make the change of variable

$$
v(x, y, \lambda)=\sum_{q=0}^{k-1} \lambda^{-q} F q(x, y) e(x, y)+\sum_{q=1}^{k} \lambda^{-q} g^{q}(x, y)+\lambda^{-k} w(x, y, \lambda), .
$$

then (2.3) takes the form

$$
\begin{aligned}
w(x, y, \lambda)= & \lambda \int_{y}^{x} R(x, t, \lambda) w(t, y, \lambda) d t+b^{0}(x, y)+d(x, y, \lambda) / \lambda \\
& +\int_{y}^{x} D(x, t, y, \lambda) e(t, y) d t+m(x, y, \lambda),
\end{aligned}
$$

where

$$
\begin{aligned}
b^{0}(x, y)= & h^{k}(x, y)-g^{k}(x, y)+\sum_{p=1}^{k} \int_{y}^{x} R^{k+1-p}(x, t) g^{p}(t, y) d t \\
& -\sum_{s=0}^{k} \sum_{p=0}^{s}(-1)^{k-s}\left[D^{k-s}\left(R^{s-p}: F^{p}\right)(x, y, y)\right] R^{0}(y, y)^{-1} e(y, y), \\
d(x, y, \lambda)= & h^{\prime}(x, y, \lambda)+\sum_{p=1}^{k} \lambda^{-(p-1)} \int_{y}^{x} R^{\prime}(x, t, \lambda) g^{p}(t, y) d t \\
& +\sum_{s=k+2}^{2 k} \sum_{p=-s-k}^{k} \lambda^{k+2-s} \int_{y}^{x} R^{s-p}(x, t) g^{p}(t, y) d t \\
D(x, t, y, \lambda)= & R^{\prime}(x, t, \lambda) \sum_{p=0}^{k} \lambda^{-p} F^{p}(t, y)
\end{aligned}
$$

$$
\begin{aligned}
& +\sum_{s=k+1}^{2 k} \sum_{p=s-k}^{k} \lambda^{k+1-s} R^{s-p}(x, t) F^{p}(t, y) \\
& +\sum_{s=0}^{k} \sum_{p=0}^{\delta}(-1)^{k+1-s} D^{k+1-s}\left(R^{s-p}: F^{p}\right)(x, t, y),
\end{aligned}
$$

$$
\begin{aligned}
m_{i}(x, y, \lambda) & =\sum_{j \neq i} \exp \left(\lambda \int_{y}^{x} \gamma_{j}(s) d s\right) \phi_{i j}(x, y) \\
\phi_{i j}(x, y) & =\gamma_{j}(x)^{-1} \sum_{s=0}^{k} \sum_{p=0}^{\dot{s}}(-1)^{k-s}\left[D^{k-s}\left(R^{s-p}: F^{p}\right)(x, x, y)\right]_{i j}
\end{aligned}
$$


The $F^{q}$ and $g^{q}$ are independent of $k$ and $\lambda$, and are determined from equations (2.18a), (2.18b), and (2.18c) (see part 2 of the proof).

Proof. In order to simplify the manipulations, we define $F^{k}(x, y) \equiv g^{0}(x, y)$ $\equiv 0$. The proof is divided into three parts.

Part 1. If $F^{q}$ and $g^{q}$ are functions on $T$ of class $C^{k+1-q}, 0 \leqq q \leqq k-1$, then under the substitution (2.7), (2.3) takes the form

$$
\begin{gathered}
\sum_{q=0}^{k} \lambda^{-q} F^{q}(x, y) e(x, y)+\sum_{q=0}^{k} \lambda^{-q} g^{q}(x, y)+\lambda^{-k} w(x, y, \lambda) \\
=\lambda^{-(k-1)} \int_{y}^{x} R(x, t, \lambda) w(t, y, \lambda) d t+\sum_{q=0}^{k} \lambda^{-q} h^{q}(x, y)
\end{gathered}
$$

$$
\begin{aligned}
& +\sum_{q=0}^{k-1} \lambda^{-q} \sum_{p=0}^{q+1} \int_{y}^{x} R^{q+1-p}(x, t) g^{p}(t, y) d t+\lambda^{-k} \sum_{p=1}^{k} \int_{y}^{x} R^{k+1-p}(x, t) g^{p}(t, y) d t \\
& +\sum_{q=0}^{k} \lambda^{-q} \sum_{s=0}^{q} \sum_{p=0}^{s}(-1)^{q-s}\left[D^{q-s}\left(R^{s-p}: F^{p}\right)(x, t, y) R^{0}(t, t)^{-1} e(t, y)\right]_{t=y}^{t=x} \\
& +\lambda^{-(k+1)} d(x, y, \lambda)+\lambda^{-k} \int_{y}^{x} D(x, t, y, \lambda) e(t, y) d t .
\end{aligned}
$$

Proof. If we substitute (2.7) in (2.3) the left side becomes the left side of (2.14), while the right side becomes

$$
\begin{aligned}
& \lambda \int_{y}^{x} \sum_{r=0}^{k} \lambda^{-r} R^{r}(x, t) \sum_{p=0}^{k} \lambda^{-p} F^{p}(t, y) e(t, y) d t \\
& +\lambda^{-k} \int_{y}^{x} R^{\prime}(x, t, \lambda) \sum_{p=0}^{k} \lambda^{-p} F^{p}(t, y) e(t, y) d t \\
& +\lambda \int_{y}^{x} \sum_{r=0}^{k} \lambda^{-r} R^{r}(x, t) \sum_{p=0}^{k} \lambda^{-p} g^{p}(t, y) d t \\
& +\lambda^{-k} \int_{y}^{x} R^{\prime}(x, t, \lambda) \sum_{p=0}^{k} \lambda^{-p} g^{p}(t, y) d t \\
& +\lambda \int_{y}^{x} \lambda^{-k} R(x, t, \lambda) w(t, y, \lambda) d t+\sum_{q=0}^{k} \lambda^{-q} h^{q}(x, y)+\lambda^{-(k+1)} h^{\prime}(x, y, \lambda) .
\end{aligned}
$$

Now for any sum we have by diagonal summation

$$
\sum_{r, p=0}^{k} \alpha_{r p}=\sum_{s=0}^{k} \sum_{p=0}^{s} \alpha_{s-p, p}+\sum_{s=k+1}^{2 k} \sum_{p=s-k}^{k} \alpha_{s-p, p .}
$$

Hence the first line of $(2.15)$ is equal to 


$$
\begin{aligned}
\sum_{s=0}^{k} \sum_{p=0}^{s} \lambda \int_{y}^{x} \lambda^{-s} R^{s-p}: F^{p}(x, t, y) e(t, y) d t \\
\quad+\sum_{s=-k+1}^{2 k} \sum_{p=-s-k}^{k} \lambda^{1-s} \int_{y}^{x} R^{s-p}: F^{p}(x, t, y) e(t, y) d t .
\end{aligned}
$$

Similarly the third line of $(2.15)$ is equal to

$$
\begin{aligned}
\sum_{s=0}^{k} \sum_{p=0}^{s} \lambda & \int_{y}^{x} \lambda^{-s} R^{s-p}: g^{p}(x, t, y) d t \\
& \quad+\sum_{s=k+1}^{2 k} \sum_{p=-\infty-k}^{k} \lambda^{1-s} \int_{y}^{x} R^{s-p}: g^{p}(x, t, y) d t .
\end{aligned}
$$

The second line of (2.15) goes into the term involving $D$ of (2.14) (see (2.11)). The fourth line and the last term go into the term involving $d$ (see (2.10)). The rest of the last line appears in the first two terms on the right of (2.14).

Since $g^{0} \equiv 0$, we may write $(2.17)$ in the form (using a change of index $q=s-1)$

$$
\begin{aligned}
& \sum_{q=0}^{k-1} \sum_{p=0}^{q+1} \lambda^{-q} \int_{y}^{x} R^{q+1-p}: g^{p}(x, t, y) d t+\sum_{p=1}^{k} \lambda^{-k} \int_{y}^{x} R^{k+1-p}: g^{p}(x, t, y) d t \\
& +\sum_{s=k+2}^{2 k} \sum_{p=s-k}^{k} \dot{\lambda}^{1-s} \int_{y}^{x} R^{s-p}: g^{p}(x, t, y) d t .
\end{aligned}
$$

The first line of $(2.17 \mathrm{a})$ is the second line of the right side of $(2.14)$, and the the second line of (2.17a) completes the term involving $d$ in (2.14).

The second line of (2.16) goes into the term involving $D$. If we apply Lemma 2.4 with $r=k+1-s$, the first line of (2.16) becomes

$$
\begin{aligned}
\sum_{s=0}^{k} \sum_{p=0}^{s} \sum_{j=0}^{k-s} & \frac{(-1)^{j}}{\lambda^{s+j}}\left[D^{i}\left(R^{s-p}: F^{p}\right)(x, t, y) R^{0}(t, t)^{-1} e(t, y)\right]_{t=y}^{l-x} \\
& +\sum_{s=0}^{k} \sum_{p=0}^{s} \frac{(-1)^{k+1-s}}{\lambda^{k}} \int_{y}^{x} D^{k+1-s}\left(R^{s-p}: F^{p}\right)(x, t, y) e(t, y) d t .
\end{aligned}
$$

The second line of (2.16a) completes the term involving $D$. If we make a change of index $q=s+j$, and interchange the $q$ and $p$ summations, the first line of $(2.16 \mathrm{a})$ becomes

$$
\sum_{s=0}^{k} \sum_{q=s}^{k} \sum_{p=0}^{s} \frac{(-1)^{q-s}}{\lambda^{q}}\left[D^{q-s}\left(R^{s-p}: F^{p}\right)(x, t, y) R^{0}(t, t)^{-1} e(t, y)\right]_{t=y}^{t=x}
$$

If we now interchange the $q$ and $s$ summations we obtain the third line on the right in (2.14).

Part 2. There exist unique functions $F^{q}, g^{q+1}$ such that for $0 \leqq q \leqq k-1$ we 
have:

(2.18a) $\quad F^{q}(x, y)=\sum_{s=0}^{q} \sum_{p=0}^{s}(-1)^{q-s} D^{q-s}\left(R^{s-p}: F^{p}\right)(x, x, y) R^{0}(x, x)^{-1}$,

(2.18b) $g^{q}(y, y)=-F^{a}(y, y) e(y, y)+h^{q}(y, y)$,

$$
\begin{aligned}
g^{q}(x, y)= & \sum_{p=0}^{q+1} \int_{y}^{x} R^{q+1-p}(x, t) g^{p}(t, y) d t+h^{q}(x, y) \\
& -\sum_{s=0}^{q} \sum_{p=0}^{s}(-1)^{q-s} D^{q-s}\left(R^{s-p}: F^{p}\right)(x, y, y) R^{0}(y, y)^{-1} e(y, y),
\end{aligned}
$$

$$
F^{q} \text { is of class } C^{k+1-q} \text {, }
$$$$
g^{q+1} \text { is of class } C^{k-q} \text {, }
$$

(2.18f) (2.18a) holds for diagonal elements when $q=k$. (Remember $F^{k}(x, y) \equiv 0$.)

Proof. For $q>0$, (2.18a) may be written as

$$
F^{q}(x, y) R^{0}(x, x)-R^{0}(x, x) F^{q}(x, y)
$$

$$
=\sum_{p=0}^{q-1} R^{q-p}(x, x) F^{p}(x, y)+\sum_{s=0}^{q-1} \sum_{p=0}^{s}(-1)^{q-s} D^{q-s}\left(R^{s-p}: F^{p}\right)(x, x, y) .
$$

The $i, j$ element on the left is $\left[\gamma_{j}(x)-\gamma_{i}(x)\right] F_{i}^{q}(x, y)$. Hence (2.18a) does not involve the diagonal elements of $F^{q}$.

Let $P_{m}$ be the proposition: There exist unique functions $F^{m}$ and $g^{m}$ such that (2.18b), (2.18c), (2.18d), and (2.18e) hold for $q=m$, while (2.18a) holds for nondiagonal elements when $q=m$, and for diagonal elements when $q=m+1$.

We first prove $\mathcal{P}_{0}$. For $q=0$, (2.18a) becomes

$$
F^{0}(x, y)=R^{0}(x, x) F^{0}(x, y) R^{0}(x, x)^{-1},
$$

or $F_{v}^{0}(x, y)=\gamma_{i}(x) F_{v}^{0}(x, y) / \gamma_{j}(x)$. This requires that we define

$$
F_{i j}^{0}(x, y)=0
$$

for $i \neq j$.

For $q=1,\left(2.18 \mathrm{a}^{\prime}\right)$ gives

$$
F^{1}(x, y) R^{0}(x, x)-R^{0}(x, x) F^{1}(x, y)=R^{1}(x, x) F^{0}(x, y)-D\left(R^{0}: F^{0}\right)(x, x, y) .
$$

For diagonal elements, the left side is 0 and we obtain the equation

$$
\begin{aligned}
{\left[D\left(R^{0}: F^{0}\right)(x, x, y)\right]_{i i} } & =D_{1} F_{i i}^{0}(x, y)-\frac{1}{\gamma_{i}(x)} F_{i i}^{0}(x, y) D_{1} R_{i i}^{0}(x, x) \\
& =R_{i i}^{1}(x, x) F_{i i}^{0}(x, y),
\end{aligned}
$$

where (2.5) and (2.20) have been used. 
For $q=0,(2.18 \mathrm{~b})$ reads

$$
-g^{0}(y, y)=0=-F^{0}(y, y) e(y, y)+h^{0}(y, y),
$$

that is,

$$
h_{i}^{0}(y, y)=F_{i i}^{0}(y, y),
$$

since $e_{i}(y, y)=1$ and $F^{0}$ is a diagonal matrix. Equations (2.21) and (2.23) determine $F_{\mathfrak{k}}^{0}(x, y)$ uniquely and $F_{\mathfrak{w}}^{0}$ is of class $C^{k+1}$ since $R^{0}$ and $h^{0}$ are of class $C^{k+1}$, and $R^{1}$ is of class $C^{k}$.

Finally (2.18c) for $q=0$ gives

$$
\begin{aligned}
g^{0}(x, y)=0= & \int_{y}^{x} R^{0}(x, t) g^{1}(t, y) d t \\
& -R^{0}(x, y) F^{0}(y, y) R^{0}(y, y)^{-1} e(y, y)+h^{0}(x, y) .
\end{aligned}
$$

By Lemma 2.5 this has a solution only if $0=-R^{0}(y, y) F^{0}(y, y) R^{0}(y, y)^{-1} e(y, y)$ $+h^{0}(y, y)$. This is satisfied because of (2.19) and (2.22). Also $g^{1}$ is of class $C^{k}$ by Lemma 2.5 since $h^{0}, F^{0}$, and $R^{0}$ are of class $C^{k+1}$.

Now let $r$ be an integer such that $1 \leqq r \leqq k-1$ and suppose $\mathcal{P}_{m}$ is true for $0 \leqq m \leqq r-1$. We shall show $\mathcal{P}_{r}$ is also true. For $q=r,\left(2.18 \mathrm{a}^{\prime}\right)$ gives

$$
\begin{aligned}
F^{r}(x, y) R^{0}(x, x)-R^{0}(x, x) F^{r}(x, y) \\
\quad=\sum_{p=0}^{r-1} R^{r-p}(x, x) F^{p}(x, y)+\sum_{s=0}^{r-1} \sum_{p=0}^{s}(-1)^{r-s} D^{r-s}\left(R^{s-p}: F^{p}\right)(x, x, y) .
\end{aligned}
$$

The $i, j$ element on the left is $\left[\gamma_{j}(x)-\gamma_{i}(x)\right] F_{v}^{r}(x, y)$. Hence for $i \neq j, F_{v}^{r}$ is uniquely determined by (2.25). By the induction hypothesis the first sum on the right of (2.25) is of class $C^{k+1-r}$, while each term in the second sum on the right is of class $C^{k+1-s-(r-s)}=C^{k+1-r}$. Hence $F_{i j}^{r}$ is of class $C^{k+1-r}$ for $i \neq j$. For $q=r+1,\left(2,18 \mathrm{a}^{\prime}\right)$ gives

$$
\begin{aligned}
F^{r+1}(x, y) & R^{0}(x, x)-R^{0}(x, x) F^{r+1}(x, y) \\
= & \sum_{p=0}^{r} R^{r+1-p}(x, x) F^{p}(x, y)+\sum_{s=0}^{r} \sum_{p=0}^{s}(-1)^{r+1-s} D^{r+1-s}\left(R^{s-p}: F^{p}\right)(x, x, y) .
\end{aligned}
$$

Equating diagonal elements, we obtain

$$
\begin{aligned}
0= & {\left[\sum_{p=0}^{r} R^{r+1-p}(x, x) F^{p}(x, y)\right]_{i i} } \\
& +\left[\sum_{i=0}^{r-1} \sum_{p=0}^{s}(-1)^{r+1-s} D^{r+1-s}\left(R^{s-p}: F^{p}\right)(x, x, y)\right]_{i i} \\
& -\left[\sum_{p=0}^{r} D\left(R^{r-p}: F^{p}\right)(x, x, y)\right]_{i i} .
\end{aligned}
$$


Upon collecting the terms involving $F^{r}$, this becomes

$$
\begin{aligned}
{\left[D\left(R^{0}: F^{r}\right)(x, x, y)\right]_{i i}-\left[R^{1}(x, x) F^{r}(x, y)\right]_{i i} } \\
=\left[\sum_{p=0}^{r-1} R^{r+1-p}(x, x) F^{p}(x, y)\right]_{i i}-\left[\sum_{p=0}^{r-1} D\left(R^{r-p}: F^{p}\right)(x, x, y)\right]_{i i} \\
\quad+\left[\sum_{s=0}^{r-1} \sum_{p=0}^{s}(-1)^{r+1-s} D^{r+1-s}\left(R^{s-p}: F^{p}\right)(x, x, y)\right]_{i i}
\end{aligned}
$$

By (2.5) the left side is

$$
\begin{aligned}
D_{1} F_{i i}^{r}(x, y) & -\frac{1}{\gamma_{i}(x)} F_{i i}^{r}(x, y) D_{1} R_{i i}^{0}(x, x)-F_{i i}^{r}(x, y) R_{i i}^{1}(x, x) \\
& +\sum_{m \neq i} F_{m i}^{r}(x, y)\left[\frac{1}{\gamma_{i}(x)} D_{2} R_{i m}^{0}(x, x)-R_{i m}^{1}(x, x)\right] .
\end{aligned}
$$

The right side of (2.26) involves previously determined functions only.

By (2.18b), for $q=r$,

$$
g^{r}(y, y)=-F^{r}(y, y) e(y, y)+h^{r}(y, y),
$$

that is,

$$
g_{i}^{r}(y, y)=-\sum_{m} F_{i m}^{r}(y, y)+h_{i}^{r}(y, y) .
$$

Since $g^{r}$ and the non-diagonal elements of $F^{r}$ have already been determined, (2.26), (2.27), and (2.29) determine $F_{4 .}^{r}$. It is of class $C^{k+1-r}$ since all the coefficients in (2.26) are of class $C^{k-r}$, and $h^{r}$ is of class $C^{k+1-r}$.

Finally by $(2.18 \mathrm{c})$ for $q=r$, we have

$$
\begin{aligned}
g^{r}(x, y)= & \sum_{p=0}^{r+1} \int_{y}^{x} R^{r+1-p}(x, t) g^{p}(t, y) d t+h^{r}(x, y) \\
& -\sum_{s=0}^{r} \sum_{p=0}^{s}(-1)^{r-s} D^{r-s}\left(R^{s-p}: F^{p}\right)(x, y, y) R^{0}(y, y)^{-1} e(y, y) .
\end{aligned}
$$

By Lemma 2.5 this will determine $g^{r+1}$ provided that

$$
\begin{aligned}
g^{r}(y, y)= & h^{r}(y, y) \\
& -\sum_{s=0}^{r} \sum_{p=0}^{s}(-1)^{r-s} D^{r-s}\left(R^{s-p}: F^{p}\right)(y, y, y) R^{0}(y, y)^{-1} e(y, y) .
\end{aligned}
$$

By (2.18a) for $q=r,(2.31)$ reduces to $g^{r}(y, y)=h^{r}(y, y)-F^{r}(y, y) e(y, y)$, which is precisely (2.28). Now $\int_{y}^{x} R^{r+1-p}(x, t) g^{p}(t, y) d t$ is of class $C^{k+1-r}$ for $1 \leqq p \leqq r$ and vanishes identically for $p=0$ since $g^{0} \equiv 0$. Also $h^{r}$ and $D^{r-s}\left(R^{s-p}: F^{p}\right), 0 \leqq p \leqq s \leqq r$, are of class $C^{k+1-r}$. Hence $g^{r+1}$ is of class $C^{k-r}$. 
By induction we have established the validity of $P_{0}, \cdots, P_{k-1}$. This proves that (2.18a) holds for nondiagonal elements when $0 \leqq q \leqq k-1$ and for diagonal elements when $1 \leqq q \leqq k$. Also (2.18a) holds for diagonal elements when $q=0$ by (2.20). The rest of part 2 also follows from $P_{0}, \cdots, P_{k-1}$.

Part 3. Using the $F^{q}, g^{q+1}$ of part 2, equation (2.3) takes the form (2.8) under the substitution (2.7).

Proof. By part 1, (2.3) takes the form (2.14). By (2.18a) and (2.18c) the coefficients of $\lambda^{-q}, 0 \leqq q \leqq k-1$, cancel (with the exception of the first term on the right). If we multiply both sides of what remains by $\lambda^{k}$ and use (2.9), we obtain (2.8) with

$$
m(x, y, \lambda)=\sum_{s=0}^{k} \sum_{p=0}^{s}(-1)^{k-s} D^{k-s}\left(R^{s-p}: F^{p}\right)(x, x, y) R^{0}(x, x)^{-1} e(x, y) .
$$

We have only to prove (2.12). By $(2.18 \mathrm{f})$, since $F^{k} \equiv 0$ and $R^{0}(x, x)^{-1}$ $=\left(\gamma_{i}(x)^{-1} \delta_{i j}\right)$,

$$
\sum_{s=0}^{k} \sum_{p=0}^{s}(-1)^{k-s}\left[D^{k-s}\left(R^{s-p}: F^{p}\right)(x, x, y)\right]_{i i}=0 .
$$

Hence

$$
\begin{aligned}
m_{i}(x, y, \lambda)= & {\left[\sum_{s=0}^{k} \sum_{p=0}^{s}(-1)^{k-s} D^{k-s}\left(R^{s-p}: F^{p}\right)(x, x, y) R^{0}(x, x)^{-1} e(x, y)\right.} \\
= & \sum_{s=0}^{k} \sum_{p=0}^{s}(-1)^{k-s} \sum_{j \neq i}\left\{\left[D^{k-s}\left(R^{s-p}: F^{p}\right)(x, x, y)\right]_{i j} \gamma_{j}(x)^{-1}\right. \\
& \left.\cdot \exp \left(\lambda \int_{y}^{x} \gamma_{j}(t) d t\right)\right\} \\
= & \sum_{j \neq i} \phi_{i j}(x, y) \exp \left(\lambda \int_{y}^{x} \gamma_{i}(t) d t\right),
\end{aligned}
$$

by (2.13).

3. The asymptotic behavior. In this section we shall assume that the expressions (2.2) and (2.4) are asymptotic expressions, that is, that there exist bounds for $K^{\prime}$ and $f^{\prime}$, and hence for $R^{\prime}$ and $h^{\prime}$ on the set $(x, y, \lambda)$ for which $(x, y) \in T, \lambda \in Z$. We shall show that these assumptions lead to bounds for the remainder $w$ in the expansion for $v$ given by Theorem 2.6.

First we define the set $Z$.

Definition 3.1. Let $Z$ be the set of points $\lambda$ in the complex plane such that

$$
R\left(\lambda \gamma_{1}(x)\right) \geqq R\left(\lambda \gamma_{i}(x)\right), \quad \quad i=2, \cdots, n ; x \in I\left(^{8}\right) .
$$

(a) $R(a)$ denotes the real part of $a$. 
If the $\gamma_{i}$ are constants there will be an ordering of the $\gamma_{i}$ for which $Z$ is not empty. Hence, since the $\gamma_{i}$ are continuous, there will be such an ordering provided $I$ is sufficiently short. This ordering is held fixed throughout this section. It is easy to prove that $Z$ is a closed sector (which may degenerate to a ray) with vertex at the origin.

Definition 3.2. Let $Z_{1}$ be the subsector of $Z$ in which $R\left(\lambda \gamma_{1}(x)\right) \leqq 0$ for all $x$ in $I$, and let $Z_{2}$ be the subsector in which $R\left(\lambda \gamma_{1}(x)\right) \geqq 0$ for all $x$ in $I$.

Definition 3.3. Let $\mathcal{T}_{i}$ be the set of points $(x, y, \lambda)$ for which $(x, y) \in T$, $\lambda \in Z_{i}, i=1,2$.

Definition 3.4. If $F$ is a function with domain $\mathcal{T}_{2}$, we shall denote by $\bar{F}$ the function on $\mathcal{T}_{2}$ such that $\bar{F}(x, y, \lambda)=e_{1}(x, y)^{-1} F(x, y, \lambda)$. A similar convention will be used for vector functions.

If $A$ is the resolvent of $F$, then it is easily seen that $\bar{A}$ will be the resolvent of $\bar{F}$. Another useful identity is $\bar{F} * \bar{G}=\overline{F * G}$.

Before finding bounds for $w$, we establish a few lemmas. We begin by considering the system

$$
w(x, y, \lambda)=\lambda \int_{y}^{x} R(x, t, \lambda) w(t, y, \lambda) d t+b(x, y, \lambda),
$$

in which $R$ and $b$ are not necessarily the same functions considered in $\$ 2$. Let $R^{0}$ be a continuous function satisfying (iii), (iv), and (v) of Hypotheses A, and let $G(x, y)=R^{0}(y, y)$. Evidently

$$
G^{*}(x, y)=\left(\frac{\gamma_{i}(y)}{p !}\left(\int_{y}^{x} \gamma_{i}(s) d s\right)^{p} \delta_{i j}\right) .
$$

If $H$ is the resolvent of $G$, it follows that $H(x, y, \lambda)=\left(\gamma_{i}(y) e_{i}(x, y) \delta_{i j}\right)$. Let

$$
\begin{aligned}
M(x, y, \lambda) & =\left(\int_{y}^{x} e_{i}(x, t) D_{1} R_{i j}^{0}(t, y) d t\right), \\
N & =M+\left(R-R^{0}\right)+\lambda H *\left(R-R^{0}\right),
\end{aligned}
$$

and let $S$ be the resolvent of $N$.

Lemma 3.5. If $R^{0}$ is of class $C^{1}$ and if $R$ and $b$ are continuous on $T$ for each $\lambda$ in $Z$, then the solution of (3.2) may be written in the form $w=p+\lambda S * p$, where $p=b+\lambda H * b$. Also $\bar{w}=\bar{p}+\lambda \bar{S} * \bar{p}$.

Proof. Let $L(x, y)=R^{0}(x, y)-R^{0}(y, y)$, and let $B=\lambda\left(R-R^{0}\right)$. Then (3.2) may be written in the form

$$
w-\lambda G * w=\lambda L * w+B * w+b .
$$

Using the fact that $H$ is the resolvent of $G$, we have

$$
w=\lambda L * w+B * w+b+\lambda^{2} H * L * w+\lambda H * B * w+\lambda H * b .
$$


But

$$
\begin{aligned}
\lambda[H * L(x, y)]_{i j} & =\lambda \int_{y}^{x} \gamma_{i}(t) e_{i}(x, t) L_{i j}(t, y) d t \\
& =-\left[L_{i j}(t, y) e_{i}(x, t)\right]_{y}^{x}+\int_{y}^{x} e_{i}(x ; t) D_{1} L_{i j}(t, y) d t \\
& =-L_{i j}(x, y)+M_{i j}(x, y, \lambda),
\end{aligned}
$$

since $L(y, y)=0, D_{1} L(t, y)=D_{1} R^{0}(t, y)$. Hence by definition of $p, B$, and $N$, $w=\lambda L * w+B * w+b-\lambda L * w+\lambda M * w+\lambda H * B * w+\lambda H * b=p+\lambda N * w$.

From this it follows that $\bar{w}=\bar{p}+\lambda \bar{N} * w \bar{w}$. Since $S$ and $\bar{S}$ are the resolvents of $N$ and $\bar{N}$ respectively, the proof of Lemma 3.5 is complete.

For the next two lemmas we make the following hypotheses about the functions in (3.2):

$$
\begin{aligned}
R(x, y, \lambda) & =R^{0}(x, y)+R^{1}(x, y) / \lambda+W(x, y, \lambda) / \lambda^{2}, \\
b(x, y, \lambda) & =b^{0}(x, y)+d(x, y, \lambda) / \lambda,
\end{aligned}
$$

where $R^{0}$ is of class $C^{2}, R^{1}$ and $b^{0}$ are of class $C^{1}$, and $W$ and $d$ are continuous on $T$ for each $\lambda$ in $Z$. As always $R^{0}(x, x)=\left(\gamma_{i}(x) \delta_{i j}\right)$.

Lemma 3.6. If $W$ is bounded in $\mathcal{T}_{1}$, then $\lambda S$ is bounded in $\mathcal{T}_{1}$. If $\bar{W}$ is bounded in $\mathcal{T}_{2}$, then $\lambda \bar{S}$ is bounded in $\mathcal{T}_{2}$.

Proof. Integrating by parts, we have

$$
\begin{aligned}
M_{i j}(x, y, \lambda)= & \int_{y}^{x} e_{i}(x, t) D_{1} R_{i j}^{0}(t, y) d t \\
= & \frac{1}{\lambda \gamma_{i}(y)} D_{1} R_{i j}^{0}(y, y) e_{i}(x, t)-\frac{1}{\lambda \gamma_{i}(x)} D_{1} R_{i j}^{0}(x, y) \\
& +\frac{1}{\lambda} \int_{y}^{x} e_{i}(x, t) \frac{\partial}{\partial t}\left[\frac{1}{\gamma_{i}(t)} D_{1} R_{i j}^{0}(t, y)\right] d t .
\end{aligned}
$$

Since $R\left(\lambda \gamma_{i}(x)\right) \leqq R\left(\lambda \gamma_{1}(x)\right) \leqq 0$, it follows that $\lambda M$ is bounded in $\mathcal{G}_{1}$. Integration by parts also shows that $\lambda H * R^{1}$ is bounded. The boundedness of $W$ implies the boundedness of $\lambda\left(R-R^{0}\right)=R^{1}+W / \lambda$ and of $H * W$. Hence $\lambda N$ is bounded in $\mathcal{G}_{1}$. If we let $\eta$ be a bound for $\lambda N$, we have

$$
\begin{aligned}
\left|N_{i j}^{2^{*}}(x, y, \lambda)\right| & \leqq \sum_{m} \int_{y}^{x}\left|N_{i m}(x, t, \lambda) N_{m j}(t, y, \lambda)\right| d t \\
& \leqq \sum_{m} \int_{y}^{x} \frac{\eta^{2}}{|\lambda|^{2}} d t=\frac{n \eta^{2}(x-y)}{|\lambda|^{2}}
\end{aligned}
$$


By induction,

$$
\left|N_{i j}^{r *}(x, y, \lambda)\right| \leqq \frac{n^{r-1} \eta^{r}(x-y)^{r-1}}{|\lambda|^{r}(r-1) !} .
$$

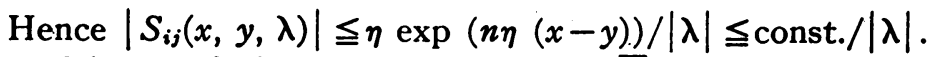

The proof of the boundedness of $\lambda \bar{N}$ in $\mathcal{T}_{2}$ proceeds as before. We have only to divide through by $e_{1}(x, y)$ after integrating by parts. The boundedness of $\lambda \bar{S}$ follows as before.

Lemma 3.7. If $W$ and $d$ are bounded in $\mathcal{T}_{1}$, then $w$ is bounded in $\mathcal{T}_{1}$. If $\bar{W}$ and $\bar{d}$ are bounded in $\mathcal{G}_{2}$, then $\bar{w}$ is bounded in $\mathcal{T}_{2}$.

Proof. Using the notation of Lemma 3.5, we have in this case

$$
\begin{aligned}
p_{i}(x, y, \lambda)= & b_{i}^{0}(x, y)+d_{i}(x, y, \lambda) / \lambda \\
& +\lambda \int_{y}^{x} \gamma_{i}(t) e_{i}(x, t)\left[b_{i}^{0}(t, y)+d_{i}(t, y, \lambda) / \lambda\right] d t .
\end{aligned}
$$

Integration by parts shows that the third term is bounded in $\mathcal{T}_{1}$, and hence $p$ is bounded in $\mathcal{G}_{1}$. Using Lemma 3.6, it follows that $\lambda S * p$ is bounded. Hence $w=p+\lambda S * p$ is bounded in $\mathcal{T}_{1}$. The proof of the second statement is similar.

LEMMA 3.8. Retaining the hypotheses on $R$ of Lemmas 3.6 and 3.7 suppose that

$$
b_{i}(x, y, \lambda)=\sum_{j \neq i} \phi_{i j}(x, y) e_{j}(x, y),
$$

where $\phi_{i j}$ is of class $C^{1}$. Then if $W$ is bounded in $\mathcal{G}_{1}, w$ is bounded in $\mathcal{G}_{1}$. If $\bar{W}$ is bounded in $\mathcal{T}_{2}, \bar{w}$ is bounded in $\mathcal{T}_{2}$.

Proof. In this case

$$
p_{i}(x, y, \lambda)=\sum_{j \neq i}\left[\phi_{i j}(x, y) e_{j}(x, y)+\lambda \int_{y}^{x} \gamma_{i}(t) e_{i}(x, t) \phi_{i j}(t, y) e_{j}(t, y) d t\right] .
$$

Now the second term in brackets is equal to

$$
\begin{aligned}
\lambda e_{i}(x, y) \int_{y}^{x} \gamma_{i}(t) \phi_{i j}(t, y) e_{j}(t, y) e_{i}(t, y)^{-1} d t & \\
= & e_{i}(x, y)\left[\frac{\gamma_{i}(t) \phi_{i j}(t, y) e_{j}(t, y) e_{i}(t, y)^{-1}}{\gamma_{j}(t)-\gamma_{i}(t)}\right]_{y}^{x} \\
& -e_{i}(x, y) \int_{y}^{x} \frac{\partial}{\partial t}\left[\frac{\gamma_{i}(t) \phi_{i j}(t, y)}{\gamma_{j}(t)-\gamma_{i}(t)}\right] e_{j}(t, y) e_{i}(t, y)^{-1} d t
\end{aligned}
$$


Hence

$$
\begin{aligned}
p_{i}(x, y, \lambda)= & \sum_{j \neq i}\left[\left(\frac{\gamma_{j}(x) \phi_{i j}(x, y)}{\gamma_{j}(x)-\gamma_{i}(x)}\right) e_{j}(x, y)-\left(\frac{\gamma_{i}(y) \phi_{i j}(y, y)}{\gamma_{j}(y)-\gamma_{i}(y)}\right) e_{i}(x, y)\right. \\
& -\int_{y}^{x} \frac{\partial}{\partial t}\left[\frac{\gamma_{i}(t) \phi_{i j}(t, y)}{\gamma_{j}(t)-\gamma_{i}(t)}\right] e_{i}(x, t) e_{j}(t, y) d t .
\end{aligned}
$$

This shows that $p$.is bounded in $\mathcal{T}_{1}$, and hence, as in Lemma 3.7, $w$ is bounded in $\mathcal{T}_{1}$. Also it is evident from (3.6) that $\bar{\phi}$, and hence $\bar{w}$, is bounded in $\mathcal{T}_{2}$.

THEOREM 3.9. In the expansion given by Theorem 2.6 for the solution of (2.3), if $R^{\prime}$ and $h^{\prime}$ are bounded in $\mathcal{T}_{1}$, then $w / \lambda$ is bounded in $\mathcal{G}_{1}$. If $\bar{R}^{\prime}$ and $\bar{h}^{\prime}$ are bounded in $\mathcal{T}_{2}$, then $\bar{w} / \lambda$ is bounded in $\mathcal{T}_{2}$.

Proof. The equation for $w$ is

$$
\begin{aligned}
w(x, y, \lambda)= & \lambda \int_{y}^{x} R(x, t, \lambda) w(t, y, \lambda) d t+b^{0}(x, y)+d(x, y, \lambda) / \lambda \\
& +\int_{y}^{x} D(x, t, y, \lambda) e(t, y) d t+m(x, y, \lambda),
\end{aligned}
$$

where $b^{0}, d, D$, and $m$ are defined in the statement of Theorem 2.6. Now $R^{q}$ and $h^{q}$ are of class $C^{k+1-q}$ by Hypotheses $A$ and we have shown that $F^{q}$ and $g^{q}$ are of class $C^{k+1-q}$. Hence for $0 \leqq p \leqq s \leqq k, R^{s-p}: F^{p}$ is of class $C^{k+1-\text { s }}$ and hence $D^{k-s}\left(R^{s-p}: F^{p}\right)$ is of class $C^{1}$. Also $R^{k+1-p}: g^{p}$ is of class $C^{1}$ for $1 \leqq p \leqq k$. Hence $b^{0}$ is of class $C^{1}$. In the same way one sees that $\phi_{i j}$ is also of class $C^{1}$ and that for each $\lambda$ in $Z, d$ and $D$ are continuous. Furthermore the hypotheses on $R^{\prime}$ and $h^{\prime}$ assure us that $d$ and $D$ are uniformly bounded. Hence $\int_{y}^{x} D(x, t, y, \lambda) e(t, y) d t$ is uniformly bounded in $\mathcal{T}_{1}$. But it does not necessarily remain bounded when multiplied by $\lambda$. Hence all we can conclude from Lemmas 3.7 and 3.8 is that $w / \lambda$ is bounded in $\tau_{1}$. The hypotheses on $\bar{R}^{\prime}$ and $\bar{h}^{\prime}$ also guarantee that $\bar{d}$ and $e_{1}(x, t)^{-1} D(x, t, y, \lambda)$ are bounded in $\tau_{2}$. Hence

$$
e_{1}(x, y)^{-1} \int_{y}^{x} D(x, t, y, \lambda) e(t, y) d t
$$

is also uniformly bounded in $\mathcal{T}_{2}$. Applying Lemmas 3.7 and 3.8 we obtain our result.

The above estimates for $w$ can be greatly improved if we restrict $\lambda$ to vary in the interior of the regions $Z_{1}, Z_{2}$. This will now be shown.

Definition 3.10. Let $Z_{1}^{\prime}$ be the subsector of $Z_{1}$ in which $\left|\pi-\arg \lambda \gamma_{1}(x)\right|$ $\leqq \pi / 2-\delta$ for all $x$ in $I$, where $\delta$ is a small positive constant whose choice will not be restricted later. Let $Z_{2}^{\prime}$ be the subsector of $Z_{2}$ in which $\left|\arg \lambda \gamma_{1}(x)\right|$ 


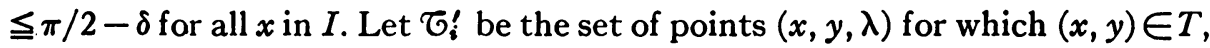
and $\lambda \in Z_{i}^{\prime}, i=1,2$.

LEMMA 3.11. Let $\psi$ be continuous for each $\lambda$ in $Z_{1}^{\prime}$ and be uniformly bounded on the set $(x, t, y, \lambda)$ for which $\alpha \leqq y \leqq t \leqq x \leqq \beta, \lambda \in Z_{1}^{\prime}$. Then

$$
\lambda \int_{y}^{x} \psi(x, t, y, \lambda) e_{i}(x, t) d t
$$

and

$$
\lambda \int_{y}^{x} \psi(x, t, y, \lambda) e_{i}(t, y) d t
$$

are uniformly bounded in $\mathcal{\sigma}_{1}^{\prime}, 1 \leqq i \leqq n$. A similar statement with $e_{i}$ replaced by $e_{i}^{-1}$ holds for $\lambda$ in $Z_{2}^{\prime}$.

Proof. Let $\eta$ be a bound for $\psi$, and let $\gamma$ be the minimum value of $\left|\gamma_{i}(x)\right|$ for $x \in I, i=1, \cdots, n$. Then $\gamma>0$ and $R\left(\lambda \gamma_{i}(x)\right) \leqq-|\lambda| \gamma \sin \delta$ in $\mathcal{T}_{1}^{\prime}$. Hence in $\mathcal{T}_{1}^{\prime}$

$$
\begin{aligned}
\left|\int_{y}^{x} \psi(x, t, y, \lambda) e_{i}(x, t) d t\right| & \leqq|\lambda| \eta \int_{y}^{x} \exp (-|\lambda| \gamma(x-t) \sin \delta) d t \\
& \leqq \frac{\eta}{\gamma \sin \delta}[1-\exp (-|\lambda| \gamma(x-y) \sin \delta] \\
& \leqq \frac{\eta}{\gamma \sin \delta} .
\end{aligned}
$$

The other statements are proved in a similar way.

For the next four lemmas we make the hypothesis that

$$
R(x, y, \lambda)=R^{0}(x, y)+W(x, y, \lambda) / \lambda,
$$

where $R^{0}$ is of class $C^{1}, W$ is continuous on $T$ for each $\lambda$ in $Z, W$ is bounded in $\mathcal{T}_{1}^{\prime}$ and $\bar{W}$ is bounded in $\mathcal{T}_{2}^{\prime}$.

LEMMA 3.12. Under the above the hypothesis, $\lambda S$ is bounded in $\mathcal{G}_{1}^{\prime}$ and $\lambda \bar{S}$ is bounded in $\mathcal{G}_{2}^{\prime}$.

Proof. In this case $N=M+W / \lambda+H * W$. Applying Lemma 3.11 we see that $\lambda N$ is uniformly bounded in $\mathcal{G}_{1}^{\prime}$. The boundedness of $\lambda S$ in $\mathcal{T}_{1}^{\prime}$ now follows by a repetition of the argument in Lemma 3.6. In the same way we prove that $\lambda \bar{S}$ is bounded in $\mathcal{T}_{2}^{\prime}$.

LemMa 3.13. Suppose that $b(x, y, \lambda)=b^{1}(x, y)+d(x, y, \lambda) / \lambda$, where $b^{0}$ is of class $C^{1}$ and $d$ is continuous on $T$ for each $\lambda$ in $Z_{1}^{\prime}$ and $d$ is bounded in $\mathcal{T}_{1}^{\prime}$. Then 


$$
w_{i}(x, y, \lambda)=b_{i}^{0}(y, y) e_{i}(x, y)+w_{i}^{\prime}(x, y, \lambda) / \lambda,
$$

where $w^{\prime}$ is bounded in $\mathcal{T}_{1}^{\prime}$.

Proof. Equation (3.4) and an integration by parts yield

$$
\begin{aligned}
p_{i}(x, y, \lambda)= & d(x, y, \lambda) / \lambda+b_{i}^{0}(y, y) e_{i}(x, y) \\
& +\int_{y}^{x} e_{i}(x, t)\left[D_{1} h_{i}^{0}(t, y)+\gamma_{i}(t) d(t, y, \lambda)\right] d t .
\end{aligned}
$$

By Lemma 3.11, $\mid p_{i}(x, y, \lambda)-\left(b_{i}^{0}(y, y) e_{i}(x, y)\left|\leqq \eta_{1} /\right| \lambda \mid\right.$, where $\eta_{1}$ is a constant. Also $\left|b_{i}^{0}(y, y) e_{i}(x, y)\right| \leqq \eta_{2} \exp (-|\lambda| \gamma(x-y)$ sin $\delta)$, where $\eta_{2}$ is a constant and $\gamma=\min \left|\gamma_{i}(x)\right|$. Hence if $\eta$ is a bound for $\lambda S$, we have

$$
\begin{aligned}
\left|\lambda S_{*} p(x, y, \lambda)_{i}\right| & =\left|\lambda \int_{y}^{x} \sum_{i} S_{i j}(x, t, \lambda) p_{i}(t, y, \lambda) d t\right| \\
& \leqq \sum_{i} \int_{y}^{x} \eta\left[\eta_{2} \exp (-|\lambda| \gamma(x-t) \sin \delta)+\eta_{1} /|\lambda|\right] d t \\
& \leqq \sum_{i} \eta\left[\frac{\eta_{2}}{|\lambda| \gamma \sin \delta}+\frac{\eta_{1}(\beta-\alpha)}{|\lambda|}\right]=\frac{\text { const. }}{|\lambda|} .
\end{aligned}
$$

Therefore $w=p+\lambda S * p$ has the stated properties.

The hypothesis on $b^{0}$ can be weakened, but the lemma does not hold. if $b^{0}$ is merely continuous or even absolutely continuous. The analogue. of Lemma 3.13 for $\lambda$ in $Z_{2}^{\prime}$ is false in general. For example the equation

$$
\theta(x)=\lambda \int_{0}^{x}\left[1-(2 / 3)(x-t)^{3 / 2}\right] \theta(t) d t+1
$$

has the solution $\theta(x)=\exp (\lambda x)\left[1-\Gamma(3 / 2) x / \lambda^{1 / 2}+\theta_{1}(x, \lambda) / \lambda\right]$ where $\theta_{1}$ is bounded for large positive $\lambda$.

LEMma 3.14. If $b(x, y, \lambda)=b^{0}(x, y)+d(x, y, \lambda) / \lambda$, where $b^{0}$ and $d$ are continuous on $T$ for each $\lambda$ in $Z_{2}^{\prime}$, while $\bar{d}$ is uniformly bounded in $\mathcal{T}_{2}^{\prime}$, then $\bar{w}$ is bounded in $\mathcal{T}_{2}^{\prime}$.

Proof. By (3.4),

$$
\begin{aligned}
\bar{p}_{i}(x, y, \lambda)= & b_{i}^{0}(x, y) e_{1}(x, y)^{-1}+\bar{d}(x, y, \lambda) / \lambda \\
& +\lambda \int_{y}^{x} \gamma_{i}(t) b_{i}^{0}(t, y) e_{i}(x, t) e_{1}(x, t)^{-1} e_{1}(t, y)^{-1} d t \\
& +\lambda \int_{y}^{x} \gamma_{i}(t) d(t, y, \lambda) e_{1}(t, y)^{-1} e_{i}(x, t) e_{1}(x, t)^{-1} d t .
\end{aligned}
$$


The first two terms on the right are bounded in $\mathcal{T}_{2}^{\prime}$, while the third term is bounded by Lemma 3.11. The last term is bounded because the integrand is bounded. Hence by Lemma 3.12, $\bar{w}=\bar{\phi}+\lambda \bar{S} * \bar{p}$ is bounded in ' $\mathcal{G}_{2}^{\prime}$.

LemMA 3.15. Let $b_{i}(x, y, \lambda)=\sum_{j \neq i} \phi_{i j}(x, y) e_{j}(x, y)$, where $\phi_{i j}$ is of class $C^{1}$. Then

$w_{i}(x, y, \lambda)=\sum_{j \neq i} \frac{\phi_{i j}(y, y)}{\gamma_{j}(y)-\gamma_{i}(y)}\left[\gamma_{j}(y) e_{j}(x, y)-\gamma_{i}(y) e_{i}(x, y)\right]+w_{i}^{\prime}(x, y, \lambda) / \lambda$, where $w^{\prime}$ is bounded in $\mathcal{T}_{1}^{\prime}$. Also $\bar{w}$ is bounded in $\mathcal{T}_{2}^{\prime}$.

Proof. In this case $p$ is given by (3.6). By Lemma 3.11 there exist constants $\eta_{1}$ and $\eta_{2}$ such that

$$
\left|p_{i}(x, y, \lambda)\right| \leqq \eta_{1} /|\lambda|+\eta_{2} \exp (-|\lambda| \gamma(x-y) \sin \delta)
$$

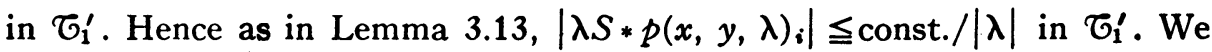
have only to show that

$$
\lambda\left[\frac{\gamma_{j}(x) \phi_{i j}(x, y)}{\gamma_{j}(x)-\gamma_{i}(x)}-\frac{\gamma_{j}(y) \phi_{i j}(y, y)}{\gamma_{j}(y)-\gamma_{i}(y)}\right] e_{j}(x, y)
$$

is uniformly bounded in $\mathcal{T}_{1}^{\prime}$. This is majorized by $|\lambda| \zeta(x-y) \exp (-|\lambda| \gamma$ $\cdot(x-y) \sin \delta)$, where

$$
\zeta=\max \left|\frac{\partial}{\partial x}\left(\frac{\gamma_{j}(x) \phi_{i j}(x, y)}{\gamma_{j}(x)-\gamma_{i}(x)}\right)\right| .
$$

When $x=y$, this is 0 , while for a fixed pair $(x, y)$ with $x>y$, its maximum as a function of $|\lambda|$ is $\zeta / e \gamma \sin \delta$. As for $w$, it is obvious from (3.6) that $\phi$ is uniformly bounded in $\mathcal{T}_{2}^{\prime}$. Hence, by Lemma 3.12, $\bar{w}=\bar{p}+\lambda \bar{S} * \bar{p}$ is bounded in $\mathcal{T}_{2}^{\prime}$.

Theorem 3.16. In the expansion given by Theorem 2.6, if $R^{\prime}$ and $h^{\prime}$ are bounded in $\mathcal{T}_{1}^{\prime}$, then

$$
\begin{aligned}
w_{i}(x, y, \lambda)= & {\left[h_{i}^{k}(y, y)-g_{i}^{k}(y, y)\right] e_{i}(x, y) } \\
& +\sum_{j \neq i} \frac{\phi_{i j}(y, y) \gamma_{j}(y)}{\gamma_{j}(y)-\gamma_{i}(y)}\left[e_{j}(x, y)-e_{i}(x, y)\right]+w_{i}^{\prime}(x, y, \lambda) / \lambda,
\end{aligned}
$$

where $w^{\prime}$ is bounded in $\mathcal{T}_{1}^{\prime}$. If $R^{\prime}$ (not just $\bar{R}^{\prime}$ ) and $\bar{h}^{\prime}$ are bounded in $\mathcal{T}_{2}^{\prime}$, then $\bar{w}$ is bounded in $\mathcal{T}_{2}^{\prime}$.

Proof. Referring to the proof of Theorem 3.9, we have seen that $\phi_{i j}$ and $b^{0}$ are of class $C^{1}$ and that for each $\lambda$ in $Z, d$ and $D$ are continuous. Also $d$ and $D$ are bounded. Hence by Lemma 3.10, $\lambda \int_{y}^{x} D(x, t, y, \lambda) e(t, y) d t$ is bounded in $\mathcal{G}_{1}^{\prime}$. By Lemmas 3.13 and 3.15 


$$
\begin{aligned}
w_{i}(x, y, \lambda)= & b_{i}^{0}(y, y) e_{i}(x, y) \\
& +\sum_{j \propto i} \frac{\phi_{i j}(y, y)}{\gamma_{j}(y)-\gamma_{i}(y)}\left[\gamma_{j}(y) e_{j}(x, y)-\gamma_{i}(y) e_{i}(x, y)\right] \\
& +w_{i}^{\prime}(x, y, \lambda) / \lambda,
\end{aligned}
$$

where $w^{\prime}$ is bounded in $\mathcal{T}_{1}^{\prime}$. But by (2.9) and (2.13),

$$
b_{i}^{0}(y, y)=h_{i}^{k}(y, y)-g_{i}^{k}(y, y)-\sum_{j \neq i} \phi_{i j}(y, y),
$$

since $\phi_{i i}(x, y) \equiv 0$ by (2.18f). As for $\bar{w}$, it is obvious that $\bar{d}$ is bounded in $\mathcal{T}_{2}^{\prime}$ and since $R^{\prime}$ is bounded in $\mathcal{T}_{2}^{\prime}, D$ is uniformly bounded. Hence by Lemma 3.10,

$$
\begin{aligned}
& \mid e_{1}(x, y)^{-1}\left(\int_{y}^{x} D(x, t, y, \lambda) e(t, y) d t\right)_{i} \mid \\
& \quad=\mid \sum_{m} \int_{y}^{x} D_{i m}(x, t, y, \lambda) e_{m}(t, y) e_{1}(t, y)^{-1} e_{1}(x, t)^{-1} d t \leqq \text { const. }|| \lambda \mid
\end{aligned}
$$

in $\mathcal{T}_{2}^{\prime}$. Applying Lemmas 3.14 and 3.15 we find that $\bar{w}$ is bounded in $\mathcal{T}_{2}^{\prime}$.

Let us examine the content of the previous theorem. Theorem 3.16 tells us that

$$
v(x, y, \lambda)=\sum_{i=0}^{k-1} \lambda^{-i} F^{i}(x, y) e(x, y)+\sum_{i=1}^{k} \lambda^{-i} g^{i}(x, y)+\lambda^{-k} w(x, y, \lambda),
$$

where $w$ is given by (3.8). Now for $\lambda$ in $Z_{1}^{\prime}$ the $F^{i}$ terms are of significance only in the neighborhood of points for which $x=y$. Indeed for a fixed point $(x, y)$ in $T$ with $x>y, \lambda^{k+1} F^{i}(x, y) e(x, y)$ and $\lambda^{k+1} w(x, y, \lambda)$ are uniformly bounded in $Z_{1}^{\prime}$. Hence we may write

$$
v(x, y, \lambda)=\sum_{i=1}^{k} \lambda^{-i} g^{i}(x, y)+r^{1}(x, y, \lambda) / \lambda^{k+1}
$$

where the remainder $r^{1}$ is bounded in $Z_{1}^{\prime}$. Similarly for a fixed point $(x, y)$ in $T$ with $x>y$ we have

$$
v(x, y, \lambda)=\sum_{i=0}^{k-1} \lambda^{-i} F^{i}(x, y) e(x, y)+r^{2}(x, y, \lambda) e_{1}(x, y) / \lambda^{k}
$$

where $r^{2}$ is bounded in $Z^{\prime}$. This shift of dominance as $\lambda$ crosses from $Z_{1}^{\prime}$ to $Z_{2}^{\prime}$ is closely analogous to Stokes' phenomenon in the theory of differential equations [ 3 , p. 554]. But the bounds on $r^{1}$ and $r^{2}$ depend on $(x, y)$ and in general become infinite as $(x, y)$ approaches the line $x=y$. Hence in order to have a remainder which is uniformly bounded in $\mathcal{T}_{1}$ and in $\mathcal{T}_{2}$, we must take into account the $F^{i}$ in the first expansion and the $g^{i}$ in the second expansion. 
However, we need only keep the part of the $F^{i}$ which represents their behavior in the neighborhood of $x=y$ well enough for our purposes. For example, if we expand $F^{0}$ in the Taylor series

$$
\begin{aligned}
F^{0}(x, y)=F^{0}(y, y)+D_{1} F^{0}(y, y)(x-y) & +\cdots \\
& +D_{1}^{k} F^{0}(y, y)(x-y)^{k} / k !+Q(x, y),
\end{aligned}
$$

then $\left|Q_{i j}(x, y)\right| \leqq \eta(x-y)^{k+1}$ in $T$, where $\eta$ is a constant. Hence by an argument analogous to the one used in Lemma 3.16, $\lambda^{k+1} Q(x, y) e(x, y)$ is uniformly bounded in $\mathcal{T}_{1}$, and $Q$ may be absorbed into $w^{\prime}$. In the same way we may replace $F^{i}(x, y)$ by the first $k+1-i$ terms in its Taylor expansion about $x=y$. For the second expansion we may replace $g^{i}$ by the first $k-i$ terms in its Taylor expansion about $x=y$.

Finally we note that Theorems 3.11 and 3.16 provided asymptotic expansions for the solutions of (2.1), since $u(x, y, \lambda)=C(x) v(x, y, \lambda)$.

4. Special cases and the resolvent kernel. In this section we shall consider cases in which certain terms of the asymptotic series for the solution of (2.3) vanish. Of special interest is the case in which $h$ is such that the solution is a column of the resolvent of $R$.

THEOREM 4.1. Suppose that $h(x, y, \lambda)=h^{0}(x, y)$. Let $p$ be a non-negative integer less than $k$. Then in the expansion (2.7), $F^{0} \equiv \ldots \equiv F^{p} \equiv 0$ if and only if $h^{0}(y, y) \equiv D_{1} h^{0}(y, y)=\cdots=D_{1}^{p} h^{0}(y, y)=0$.

Proof. If $h_{\mathbf{a}}^{0}(y, y) \equiv 0$, then $F_{0}^{\mathfrak{a}}(y, y) \equiv 0$ by $(2.23)$. Hence by $(2.21)$, $F_{\mathrm{st}}^{0}(x, y) \equiv 0$. Also the nondiagonal elements of $F^{0}$ are already identically 0 . By (2.24)

$$
\int_{y}^{x} R^{0}(x, t) g^{1}(t, y) d t+h^{0}(x, y)=0
$$

Hence

$$
D_{1} h^{0}(x, y)=-R^{\mathrm{c}}(x, x) g^{1}(x, y)+\int_{y}^{x} D_{1} R^{0}(x, t) g^{1}(t, y) d t,
$$

and therefore $0=D_{1} h^{0}(y, y)=R^{0}(y, y) g^{1}(y, y)$. Since $R^{0}(y, y)$ is nonsingular, it follows that $g^{1}(y, y) \equiv 0$. In the same way one sees that $D_{1}^{i} g^{1}(y, y)=0$ for $1 \leqq i \leqq p-1$. Let $r$ be a positive integer less than $p$, and suppose that for $1 \leqq j \leqq r$ we have $F^{i-1}(x, y) \equiv 0$ and $g^{j}(y, y) \equiv D_{1} g^{j}(y, y)=\cdots=D_{1}^{p-j} g^{i}(y, y)$ $\equiv 0$. By (2.25), $F^{r}(x, y) R^{0}(x, x)-R^{0}(x, x) F^{r}(x, y)=0$. Hence the nondiagonal elements of $F^{r}$ are identically 0 . Also by $(2.26)$ and (2.27), $F_{t z}^{r}$ satisfies a homogeneous first order linear differential equation. But by $(2.29)$ and the induction hypothesis $0 \equiv g_{i}^{r}(y, y)=F_{\mathfrak{t}}^{r}(y, y)$. Hence the diagonal elements of $F^{r}$ also vanish identically. Finally by $(2.30)$ 


$$
g^{r}(x, y)=\sum_{p=0}^{r+1} \int_{y}^{x} R^{r+1-p}(x, t) g^{p}(t, y) d t .
$$

If we differentiate $p-r$ times we find that $g^{r+1}(y, y) \equiv D_{1} g^{r+1}(y, y)=\ldots$ $\equiv D_{1}^{p-r-1} g^{r+1}(y, y)$. This completes our induction proof of the first part of our statement.

Now suppose $F^{0} \equiv \cdots \equiv F^{p} \equiv 0$. Then by (2.18c) we have

$$
\begin{aligned}
-h^{0}(x, y) & =\int_{y}^{x} R^{0}(x, t) g^{1}(t, y) d t, \\
g^{1}(x, y) & =\int_{y}^{x} R^{0}(x, t) g^{2}(t, y) d t+\int_{y}^{x} R^{1}(x, t) g^{1}(t, y) d t, \\
\ldots & \ldots \ldots \ldots \\
g^{p}(x, y) & =\int_{y}^{x} R^{0}(x, t) g^{p+1}(t, y) d t+\ldots \ldots+\int_{y}^{x} R^{p}(x, t) g^{1}(t, y) d t .
\end{aligned}
$$

Hence $h^{0}(y, y)=g^{1}(y, y)=\cdots=g^{p}(y, y)=0$. If we differentiate the first $p$ equations we find that $D_{1} h^{0}(y, y)=D_{1} g^{1}(y, y)=\cdots=D_{1} g^{p-1}(y, y)=0$. Continuing in this way we arrive at our result.

In the general case where the $h^{q}$ are not identically 0 for $q \geqq 1$, the condition that $F^{0} \equiv \cdots \equiv F^{p} \equiv 0$ becomes $g^{i}(y, y)=h^{i}(y, y), j=0, \cdots, p$. This leads to a complicated condition on the values of the $h^{q}$ and their derivatives at $x=y$.

We now turn our attention to the $g$ 's. The case in which $g^{1}$ is identically 0 turns out to be equivalent to the case in which the solution of (2.3) is a column of the resolvent of $R$.

THEOREM 4.2. In the expansion (2.7), $g^{1} \equiv 0$ if and only if $h^{0}(x, y)$ $=R^{0}(x, y) b(y)$ for some vector $b$ depending on $y$ alone.

Proof. By (2.24), $g^{1} \equiv 0$ if and only if

$$
h^{0}(x, y)=R^{0}(x, y) F^{0}(y, y) R^{0}(y, y)^{-1} e(y, y) .
$$

Hence if $g^{1} \equiv 0, h^{0}$ must have the form indicated. Conversely if $h^{0}(x, y)$ $=R^{0}(x, y) b(y)$, then $b(y)=R^{0}(y, y)^{-1} h^{0}(y, y)$. Hence

$$
h^{0}(x, y)=R^{0}(x, y) R^{0}(y, y)^{-1} h^{0}(y, y)=R^{0}(x, y) R^{0}(y, y)^{-1} F^{0}(y, y) e(y, y)
$$

by (2.22), and the right side is equal to $R^{0}(x, y) F^{0}(y, y) R^{0}(y, y)^{-1} e(y, y)$ since diagonal matrices commute.

Definition 4.3. Let $e^{p}$ be the vector whose $i$ th component is $\delta_{i p}$.

If we set $h=R e^{p}$, then $h$ becomes the pth column of $R$. In this case the solution of (2.3) will be the $p$ th column of the resolvent of $R$ (see $\$ 1$ ). Thus Theorem 2.6 furnishes an asymptotic expansion for each column of the resolvent. By Theorem 4.2, in each of these expansions $g^{1} \equiv 0$. A natural 
question then is the nature of kernels $R$ which are such that $g^{2} \equiv 0$ in the expansion for each of the columns of the resolvent. This is answered by:

THEOREM 4.4. In the expansions for the columns of the resolvent $S$ of $R$ all the $\mathrm{g}^{1}$ 's are identically 0 , and all the $\mathrm{g}^{2}$ 's are identically 0 if and only if $R^{0}(x, y)$ has the form $A(x) B(y)$.

Proof. In (2.7) we set $h^{i}=R^{i} e^{p}$. Then $g^{1} \equiv 0$ and the equation for $g^{2}$ becomes

$$
\begin{aligned}
0= & \int_{y}^{x} R^{0}(x, t) g^{2}(t, y) d t+h^{1}(x, y) \\
& +\left[D\left(R^{0}: F^{0}\right)(x, y, y)-R^{1}(x, y) F^{0}(y, y)\right. \\
& \left.-R^{0}(x, y) F^{1}(y, y)\right] R^{0}(y, y)^{-1} e(y, y) .
\end{aligned}
$$

By (2.22) we have

$$
\begin{aligned}
& R^{1}(x, y) F^{0}(y, y) R^{0}(y, y)^{-1} e(y, y) \\
& \quad=R^{1}(x, y) R^{0}(y, y)^{-1} h^{0}(y, y)=R^{1}(x, y) e^{p}=h^{1}(x, y)
\end{aligned}
$$

since the diagonal matrices $F^{0}(y, y)$ and $R^{0}(y, y)^{-1}$ commute. Also by (2.18a) $F^{1}(y, y)=\left[-D\left(R^{0}: F^{0}\right)(y, y, y)+R^{1}(y, y) F^{0}(y, y)+R^{0}(y, y) F^{1}(y, y)\right] R^{0}(y, y)^{-1}$.

Hence if we multiply each side on the right by $e(y, y)$ and use $(2.18 \mathrm{~b})$ and (4.2) we obtain

$h^{1}(y, y)=\left[-D\left(R^{0}: F^{0}\right)(y, y, y)+R^{0}(y, y) F^{1}(y, y)\right] R^{0}(y, y)^{-1} e(y, y)+h^{1}(y, y)$.

Thus

$$
\begin{aligned}
& R^{0}(x, y) F^{1}(y, y) R^{0}(y, y)^{-1} e(y, y) \\
& \quad=R^{0}(x, y) R^{0}(y, y)^{-1} D\left(R^{0}: F^{0}\right)(y, y, y) R^{0}(y, y)^{-1} e(y, y) .
\end{aligned}
$$

Now (4.1), (4.2), and (4.3) show that $g^{2} \equiv 0$ in the expansion for the $p$ th column of $S$ if and only if

$$
\begin{aligned}
& {\left[D\left(R^{0}: F^{0}\right)(x, y, y)\right.} \\
& \left.\quad-R^{0}(x, y) R^{0}(y, y)^{-1} D\left(R^{0}: F^{0}\right)(y, y, y)\right] R^{0}(y, y)^{-1} e(y, y)=0 .
\end{aligned}
$$

By definition

$$
\begin{aligned}
D\left(R^{0}: F^{0}\right)(x, y, y)= & \frac{\partial}{\partial t}\left[R^{0}(x, t) F^{0}(t, y) R^{0}(t, t)^{-1}\right]_{t=y} \\
= & R^{0}(x, y) \frac{\partial}{\partial t}\left[F^{0}(t, y) R^{0}(t, t)^{-1}\right]_{t=y} \\
& +D_{2} R^{0}(x, y) F^{0}(y, y) R^{0}(y, y)^{-1}
\end{aligned}
$$


Hence

$$
\begin{aligned}
D\left(R^{0}: F^{0}\right)(y, y, y)= & R^{0}(y, y) \frac{\partial}{\partial t}\left[F^{0}(t, y) R^{0}(t, t)^{-1}\right]_{t=y} \\
& +D_{2} R^{0}(y, y) F^{0}(y, y) R^{0}(y, y)^{-1} .
\end{aligned}
$$

Thus the bracketed expression in (4.4) reduces to

$$
\left[D_{2} R^{0}(x, y)-R^{0}(x, y) R^{0}(y, y)^{-1} D_{2} R^{0}(y, y)\right] F^{0}(y, y) R^{0}(y, y)^{-1} .
$$

Therefore our condition becomes

$$
\left[D_{2} R^{0}(x, y)-R^{0}(x, y) R^{0}(y, y)^{-1} D_{2} R^{0}(y, y)\right] R^{0}(y, y)^{-1} e^{p}=0 .
$$

Since $R^{0}(y, y)^{-1}$ is nonsingular, this holds for $p=1, \cdots, n$ if and only if

$$
D_{2} R^{0}(x, y)=R^{0}(x, y) R^{0}(y, y)^{-1} D_{2} R^{0}(y, y) \text {. }
$$

Our proof will now be complete if we apply the following lemma.

LeMma 4.5. Let $F$ be a function matrix of class $C^{1}$ on $T$ and suppose $F(y, y)$ is nonsingular. $A$ necessary and sufficient condition that $F(x, y)$ be expressible in the form $A(x) B(y)$ is that $D_{2} F(x, y)=F(x, y) F(y, y)^{-1} D_{2} F(y, y)$.

Proof. If $F(x, y)=A(x) B(y)$, then $A$ and $B$ are nonsingular and it is easily seen that the condition is satisfied. Conversely, if $D_{\mathbf{2}} F(x, y)$ $=F(x, y) C(y)$, then $F$ satisfies the system

$$
\frac{d}{d y} Y(y)=Y(y) C(y)
$$

with initial conditions $Y(x)=F(x, x)$. Now let $Y_{1}(y)$ be a particular solution of this system with $Y_{1}(\alpha)$ nonsingular. Then by a classical theorem $Y_{1}(y)$ is nonsingular in $I$. Hence for each $x$ in $I, F(x, y)=F(x, x) Y_{1}(x)^{-1} Y_{1}(y)$ for $\alpha \leqq y \leqq x$ by the uniqueness theorem.

THEOREM 4.6. If $R(x, y, \lambda)=R^{0}(x, y)=A(x) B(y)$, then in the expansion for the columns of $S$ all the $g$ 's are identically 0 .

Proof. In this case $D_{2} R^{0}(x, y)=R^{0}(x, y) C^{1}(y)$, and hence $D_{2}^{a} R^{0}(x, y)$ $=R^{0}(x, y) C^{q}(y)$. Now

$$
\begin{aligned}
D\left(R^{0}: F^{j}\right)(x, y, y)= & D_{2} R^{0}(x, y) F^{i}(y, y) R^{0}(y, y)^{-1} \\
& +R^{0}(x, y) \frac{\partial}{\partial t}\left[F^{i}(t, y) R^{0}(t, t)^{-1}\right]_{t=y} .
\end{aligned}
$$

By (4.5) the right side has the form $R^{0}(x, y) P^{1 j}(y)$, and similarly

$$
D^{i}\left(R^{0}: F^{i}\right)(x, y, y)=R^{0}(x, y) P^{i j}(y) \text {. }
$$


Hence

$$
D^{i}\left(R^{0}: F^{j}\right)(x, y, y)=R^{0}(x, y) R^{0}(y, y)^{-1} D^{i}\left(R^{0}: F^{j}\right)(y, y, y) .
$$

Now suppose all the $g^{2}$ s, $\cdots, g^{r}$ s are identically $0, r \geqq 2$. Then by $(2.18 \mathrm{c})$ the $g^{r+1}$ for the pth column of $S$ is identically 0 if and only if

$$
\sum_{s=0}^{r}(-1)^{r-s} D^{r-s}\left(R^{0}: F^{s}\right)(x, y, y) R^{0}(y, y)^{-1} e(y, y)=0,
$$

since in our case $h^{q} \equiv 0$ for $q>0$. Now by (2.18a) we have

$$
F^{r}(y, y)=\sum_{s=0}^{r}(-1)^{r-s} D^{r-s}\left(R^{0}: F^{s}\right)(y, y, y) R^{0}(y, y)^{-1} .
$$

Hence by (2.18b)

$$
\sum_{s=0}^{r}(-1)^{r-s} D^{r-s}\left(R^{0}: F^{s}\right)(y, y, y) R^{0}(y, y)^{-1} e(y, y)=0 .
$$

Therefore

$$
\begin{aligned}
F^{r}(y, y) R^{0}(y, y)^{-1} e(y, y) \\
\quad=-R^{0}(y, y)^{-1} \sum_{s=0}^{r-1}(-1)^{r-s} D^{r-s}\left(R^{0}: F^{s}\right)(y, y, y) R^{0}(y, y)^{-1} e(y, y) .
\end{aligned}
$$

Substituting this in the $s=r$ term of (4.7) we obtain

$$
\begin{aligned}
0= & {\left[\sum_{s=0}^{r-1}(-1)^{r-s} D^{r-s}\left(R^{0}: F^{s}\right)(x, y, y)\right.} \\
& \left.-R^{0}(x, y) R^{0}(y, y)^{-1} \sum_{s=0}^{r-1}(-1)^{r-s} D^{r-s}\left(R^{0}: F^{s}\right)(y, y, y)\right] R^{0}(y, y)^{-1} e(y, y) .
\end{aligned}
$$

But the expression in brackets vanishes because of (4.6). The case $r=2$ has been covered in Theorem 4.4.

In the general case the situation is described in the next theorem. The proof is long and will be omitted.

THEOREM 4.7. In the expansion for the columns of $S$, all the $g^{2 '} s, \cdots, g^{r} s$ are identically 0 if and only if there exist matrices $A^{0}, \cdots, A^{r-2}, B^{0}, \cdots, B^{r-2}$ such that

$$
\begin{aligned}
& R^{0}(x, y)=A^{0}(x) B^{0}(y), \\
& R^{1}(x, y)=A^{0}(x) B^{1}(y)+A^{1}(x) B^{0}(y), \\
& R^{r-2}(x, y)=A^{0}(x) B^{r-2}(y)+\cdots+A^{r-2}(x) B^{0}(y) .
\end{aligned}
$$


As an illustration of Theorems 4.4 and 4.6, consider the case in which

$$
R(x, y, \lambda)=R^{0}(x, y)=\left(\begin{array}{cc}
1 & x-y \\
0 & x
\end{array}\right)
$$

and $T$ does not contain the origin. It is easily seen that $R^{0}(x, y)^{-1} D_{2} R^{0}(x, y)$ depends on $y$ alone, and hence $R^{0}(x, y)$ is expressible in the form $A(x) B(y)$. For example we may choose

$$
A(x)=\left(\begin{array}{ll}
x & 1 \\
x & 0
\end{array}\right), \quad B(y)=\left(\begin{array}{rr}
0 & 1 \\
1 & -y
\end{array}\right) .
$$

In this case $S$ is easily found by solving the second equation of the system first.

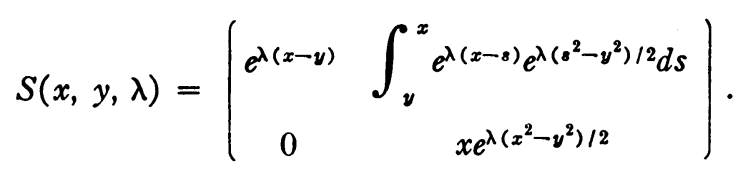

The asymptotic expansion of $S_{12}(x, y, \lambda)$ consists of a sum of two series, one multiplied by exp $(\lambda(x-y))$ and the other by exp $\left(\lambda\left(x^{2}-y^{2}\right) / 2\right)$. Note that when $R(x, y, \lambda)=A(x) B(y)$, it is by no means always possible to obtain simple explicit expressions for $S(x, y, \lambda)$ (except when $n=1$ ).

The solution $u$ of (2.1) has the asymptotic expansion

$$
\begin{aligned}
u(x, y, \lambda)= & \sum_{i=0}^{k-1} \lambda^{-i} C(x) F^{i}(x, y) e(x, y) \\
& +\sum_{i=1}^{k} \lambda^{-i} C(x) g^{i}(x, y)+\lambda^{-k} C(x) w(x, y, \lambda) .
\end{aligned}
$$

Now $h(x, y, \lambda)=C(x)^{-1} f(x, y, \lambda)$ and $R(x, y, \lambda)=C(x)^{-1} K(x, y, \lambda) C(y)$. Hence in the statements of Theorems 4.1 and 4.2, we may replace $h$ and $R$ by $f$ and $K$. As for the other theorems in this section, let $Q$ be the resolvent of $K$. It is easily seen from the relations $Q-K=\lambda K * Q, S-R=\lambda R * S$, that $S(x, y, \lambda)$ $=C(x)^{-1} Q(x, y, \lambda) C(y)$. Hence the $g^{2}$ 's for all the columns of $Q$ are identically 0 if and only if the $g^{2}$ 's for all the columns of $S$ are identically 0 . Also $R^{0}(x, y)=A(x) B(y)$ if and only if $K^{0}(x, y)=C(x) A(x) B(y) C(y)^{-1}$. Hence in Theorems 4.4 and 4.6 we need only replace $S$ and $R$ by $Q$ and $K$. The same applies to the statement of Theorem 4.7.

5. An application. The results of the previous sections may be applied to give the asymptotic behavior of solutions to integro-differential equations of the form

$$
\begin{aligned}
\theta^{(n-1)}(x, \lambda)+\lambda \alpha_{1}(x, \lambda) \theta^{(n-2)}(x, \lambda) & +\cdots+\lambda^{n-1} \alpha_{n-1}(x, \lambda) \theta(x, \lambda) \\
& +\lambda^{n} \int_{\alpha}^{x} \Gamma(x, t, \lambda) \theta(t, \lambda) d t=\lambda^{n} \phi(x, \lambda) .
\end{aligned}
$$


where $\theta^{(k)}(x, \lambda)=d^{k} / d x^{k} \theta(x, \lambda)$, and the given functions have asymptotic developments of the form

$$
\begin{aligned}
\alpha_{i}(x, \lambda) & =\alpha_{i}^{0}(x)+\alpha_{i}^{1}(x) / \lambda+\cdots \\
\phi(x, \lambda) & =\phi^{0}(x)+\phi^{1}(x) / \lambda+\cdots \\
\Gamma(x, y, \lambda) & =\Gamma^{0}(x, y)+\Gamma^{1}(x, y) / \lambda+\cdots .
\end{aligned}
$$

If we set

$$
u_{i}(x, \lambda)=\lambda^{-i \theta^{(i-1)}}(x, \lambda), \quad i=1, \cdots, n,
$$

then for $1 \leqq i \leqq n-1$, we have

$$
u_{i}(x, \lambda)=\lambda \int_{\alpha}^{x} u_{i+1}(t, \lambda) d t+\lambda^{-i} \theta^{(i-i)}(\alpha, \lambda),
$$

and by (5.1)

$$
\begin{aligned}
& u_{n}(x, \lambda)+\alpha_{1}(x, \lambda) u_{n-1}(x, \lambda)+\cdots+\alpha_{n-1}(x, \lambda) u_{1}(x, \lambda) \\
& \quad+\lambda \int_{\alpha}^{x} \Gamma(x, t, \lambda) u(t, \lambda) d t=\phi(x, \lambda) .
\end{aligned}
$$

Using (5.3) we have

$$
\begin{aligned}
u_{n}(x, \lambda) & +\lambda \int_{\alpha}^{x} \alpha_{1}(x, \lambda) u_{n}(t, \lambda) d t+\cdots+\lambda \int_{\alpha}^{x} \alpha_{n-1}(x, \lambda) u_{2}(t, \lambda) d t \\
& +\lambda \int_{\alpha}^{x} \Gamma(x, t, \lambda) u_{1}(t, \lambda) d t \\
& =\phi(x, \lambda)-\alpha_{n-1}(x, \lambda) \theta(\alpha, \lambda) / \lambda-\cdots-\alpha_{1}(x, \lambda) \theta^{(n-2)}(\alpha, \lambda) / \lambda^{n-1} .
\end{aligned}
$$

Equations (5.3) and (5.4) form a system of integral equations with kernel matrix

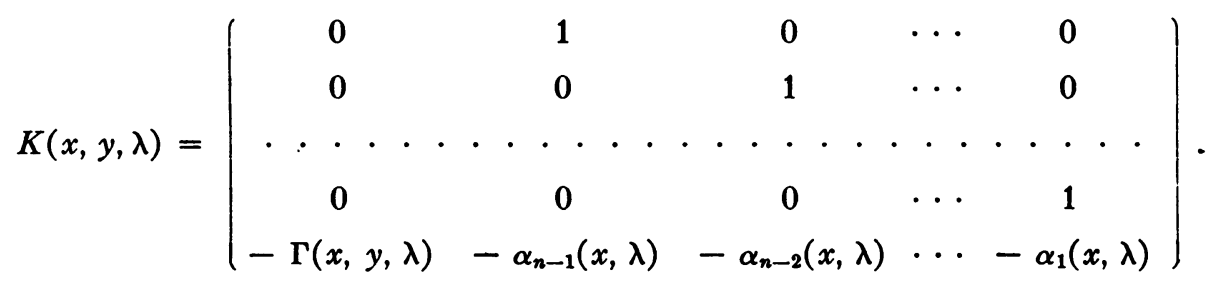

The characteristic equation for $K^{0}(x, x)$ is

$$
\rho^{n}+\rho^{n-1} \alpha_{1}^{0}(x)+\cdots+\rho \alpha_{n-1}^{0}(x)+\Gamma^{0}(x, x)=0 .
$$

If the roots of this equation are distinct and diierent from 0 for all $x$ in $I$ 
we may apply our theory to this system.

Consider the single equation

$$
\theta(x)=\mu \int_{\alpha}^{x} \Gamma(x, t) \theta(t) d t+\mu \phi(x) .
$$

As $\mu \rightarrow \infty,(5.5)$ reduces to an integral equation of the first kind

$$
\int_{\alpha}^{x} \Gamma(x, t) \theta(t) d t+\phi(x)=0 .
$$

As an application of our theory we shall investigate the convergence of the solution of (5.5) to the solution of (5.6). Peres [4, pp. 26-30] has shown that if $\Gamma$ and $\phi$ are real-valued and of class $C^{2}$, and if $\Gamma(x, x)$ is positive in $I$, then the solution of (5.5) approaches the solution of (5.6) as $\mu \rightarrow \infty$ along the negative real axis (provided that $\phi(\alpha)=0$, so that (5.6) has a solution).

We suppose that $\Gamma$ is real-valued and is of order $n$. That is, there exists an integer $n \geqq 1$ such that $D_{1}^{n-1} \Gamma(x, x) \neq 0$ in $I$ and $D_{1}^{p} \Gamma(x, x) \equiv 0$ for $0 \leqq p$ $<n-1$. By adjusting the sign of $\mu$ we may suppose that $\Lambda(x)=D_{1}^{n-1} \Gamma(x, x)$ is positive in $I$. In order that (5.6) have a solution, we assume that $\phi(\alpha)=\cdots=\phi^{(n-1)}(\alpha)=0$.

Now (5.5) is equivalent to the integro-differential equation

$$
\theta^{(n-1)}(x)=\mu \int_{\alpha}^{x} D_{1}^{n-1} \Gamma(x, t) \theta(t) d t+\mu \phi^{(n-1)}(x)
$$

with initial conditions $\theta(\alpha)=\theta^{(1)}(\alpha)=\cdots=\theta^{(n-2)}(\alpha)=0$. If we make the substitution (5.2) where $\lambda^{n}=\mu$ we find that (5.7) is equivalent to the system

$$
u(x)=\lambda \int_{\alpha}^{x} K(x, t) u(t) d t+f(x),
$$

where

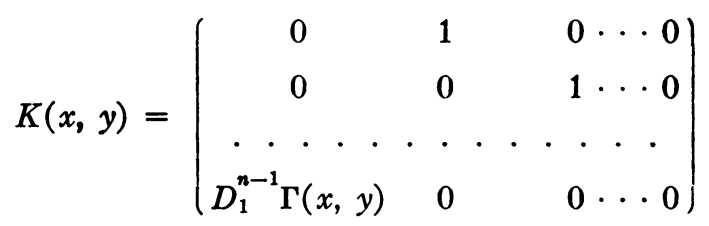

and

$$
f_{i}(x)=\delta_{i n} \phi^{(n-1)}(x) .
$$

By hypothesis $f(\alpha)=0$. The characteristic equation for $K(x, x)$ is $\rho^{n}-\Lambda(x)=0$. If $\gamma_{1}(x), \cdots, \gamma_{n}(x)$ is a certain ordering of the roots, then under the substitution described in $\$ 2$, we obtain the equation 


$$
v(x)=\lambda \int_{\alpha}^{x} R(x, t) v(t) d t+h(x) .
$$

In the expansion given by Theorem 2.1 we shall drop the letter indicating the dependence of the coefficients on the lower limit of integration. Now $h(\alpha)=C(\alpha)^{-1} f(\alpha)=0$. Hence by Theorem $4.1, F^{0}(x) \equiv 0$. By (2.24) the equation for $g^{1}$ is

$$
0=\int_{\alpha}^{x} R(x, t) g^{1}(t) d t+h(x) .
$$

If we multiply through on the right by $C(x)$ we obtain

$$
\int_{\alpha}^{x} K(x, t)\left[C(t) g^{1}(t)\right] d t+f(x)=0 .
$$

Using (5.8) and (5.9) we find that all the components of $C(x) g^{1}(x)$ are 0 except for the first. If we denote the first component of $C(x) g^{1}(x)$ by $\xi(x)$, we have

$$
\int_{\alpha}^{x} D_{1}^{n-1} \Gamma(x, t) \xi(t) d t+\phi^{(n-1)}(x)=0 .
$$

Now (5.10) shows that $\xi$ is the solution of (5.6).

If $\phi^{(n)}(\alpha)=0$, then $h^{(1)}(\alpha)=0$ and hence, by Theorem $4.1, F^{1}(x) \equiv 0$. Conversely if $\phi^{(n)}(\alpha) \neq 0$, not all the elements in the first row of $C(\alpha) F^{1}(\alpha)$ are 0 . Indeed their sum is $\left[C(\alpha) F^{1}(\alpha) e(\alpha)\right]_{1}=-\left[C(\alpha) g^{1}(\alpha)\right]_{1}=-\xi(\alpha)$ by $(2.18 \mathrm{~b})$, and from (5.10), $-\xi(\alpha)=\phi^{(n)}(\alpha) / \Lambda(\alpha)$.

Now suppose $\Gamma$ and $\phi$ are of class $C^{n+3}$, Applying Theorem 3.10 with $k=3$, we have

$$
u(x)=C(x) F^{1}(x) e(x) / \lambda+C(x) g^{1}(x) / \lambda+r(x, \lambda) / \lambda^{2},
$$

where $r(x, \lambda)=g^{2}(x)+g^{3}(x) / \lambda+F^{2}(x) e(x)+w(x, \lambda) / \lambda$ is bounded in $\mathcal{T}_{1}$, and $\bar{r}$ is bounded in $\mathcal{G}_{2}$. If we multiply by $\lambda$ and equate first components, we have

$$
\theta(x)=\lambda u_{1}(x)=\sum_{j}\left[C(\alpha) F^{1}(\alpha)\right]_{1 j} e_{j}(x)+\xi(x)+r_{1}(x, \lambda) / \lambda .
$$

Thus if $\phi^{(n)}(\alpha)=0, F^{1}(\dot{\alpha})=0$ and $\theta(x)$ approaches $\xi(x)$ uniformly in $I$ as $\lambda \rightarrow \infty$ in $Z_{1}$, and the same will be true if $\lambda \rightarrow \infty$ in $Z_{2}$ in such a way that $R\left(\lambda \gamma_{1}(x)\right)$ remains bounded above. If $\phi^{(n)}(\alpha) \neq 0, \theta(x)$ does not approach $\xi(x)$ as $\lambda \rightarrow \infty$ in $Z_{1}$, but it does for $\lambda$ in $Z_{1}^{\prime}$, and the convergence will be uniform in any interval of the form $\alpha+\eta \leqq x \leqq \beta$, where $\eta$ is a small positive constant. Also $\lim \sup |\theta(x)|=+\infty$ as $\lambda \rightarrow \infty$ in $Z_{2}$ in such a way that $R\left(\lambda \gamma_{1}(x)\right) \rightarrow+\infty$.

Now for $n=1$, the $Z_{1}$ region is the closed left half-plane, the $Z_{1}^{\prime}$ region is the sector $|\pi-\arg \mu| \leqq \pi / 2-\delta$, and the $Z_{2}$ region is the closed right half-plane. 
Hence if $\phi^{(1)}(\alpha)=0, \theta(x) \rightarrow \xi(x)$ uniformly in $I$ as $\mu \rightarrow \infty$ in such a way that $R(\mu)$ remains bounded above. If $\phi^{(1)}(\alpha) \neq 0$, then $\theta(x) \rightarrow \xi(x)$ uniformly in $\alpha+\eta \leqq x \leqq \beta$ as $\mu \rightarrow \infty$ in the sector $|\pi-\arg \mu| \leqq \pi / 2-\delta$. This last statement can also be proved by using Theorem 3.14 if $\Gamma$ and $\phi$ are only of class $C^{2}$.

For $n>1$ we need only consider the $Z$ regions lying in the sector $0 \leqq \arg \lambda$ $\leqq 2 \pi / n$ since the solution of.(5.5) is single-valued in $\mu$.

For $n=2$, the $Z$ region corresponding to the choice $\gamma_{1}(x)=+(\Lambda(x))^{1 / 2}$ is the closed left half-plane and it is a $Z_{2}$ region as well. Also the $Z$ region corresponding to the choice $\gamma_{1}(x)=-(\Lambda(x))^{1 / 2}$ is the closed right half-plane and it is a $Z_{2}$ region as well. The $Z_{1}$ region in each case is the imaginary axis. Hence if $\phi^{(2)}(\alpha)=0, \theta(x)$ approaches $\xi(x)$ uniformly in $I$ as $\lambda \rightarrow \infty$ in such a way that $|R(\lambda)|$ remains bounded, that is, as $\mu \rightarrow \infty$ in such a way that $\left|R\left(\mu^{1 / 2}\right)\right|$ remains bounded. If $\phi^{(1)}(\alpha) \neq 0$, then $\lim \sup |\theta(x)|=\infty$ as $\lambda \rightarrow \infty$ in such a way that $R(\lambda) \rightarrow \pm \infty$.

Finally for $n \geqq 3$ the sectors $0 \leqq \arg \lambda \leqq \pi / n, \pi / n \leqq \arg \lambda \leqq 2 \pi / n$ are $Z$ regions and $Z_{2}$ regions as well. In each of these sectors

$$
R\left(\lambda \gamma_{1}(x)\right)=\max _{1 \leqq i \leqq n} R\left(\lambda \gamma_{i}(x)\right) \geqq \frac{|\lambda|}{2}(\Lambda(x))^{1 / n} .
$$

Hence $R\left(\lambda \gamma_{1}(x)\right) \rightarrow+\infty$ as $\lambda \rightarrow \infty$. Thus if $\Gamma$ and $\phi$ are differentiable infinitely of ten, the only possibility for $\lim \sup |\theta(x)|$ to remain finite as $\mu \rightarrow \infty$ is for all derivatives of $\phi$ to be 0 at $x=\alpha$.

\section{REFERENCES}

1. G. D. Birkhoff and R. E. Langer, The boundary problems and developments associated with a system of ordinary linear differential equations of the first order, Proceedings of the American Academy of Arts and Sciences vol. 58 (1923) pp. 51-128.

2. G. Kowalewski, Integralgleichungen, Berlin, 1930.

3. R. E. Langer, The asymptotic solutions of ordinary linear differential equations of the second order, with special reference to the Stokes phenomenon, Bull. Amer. Math. Soc. vol. 40 (1934) pp. 545-582.

4. J. Peres, Sur les transformations qui conservent la composition, Bull. Soc. Math. France vol. 47 (1919) pp. 16-37.

5. W. J. Trjitzinsky, Theory of linear differential equations containing a parameter, Acta Math. vol. 67 (1936) pp. 1-50.

6. V. Volterra, Teoria delle potenze, dei logaritmi e delle funzione di composizione, Memoire della Reale Accademia dei Lincei (5) vol. 11 (1915) pp. 167-249.

University of California, Berkeley, Calif. 